(c) 2004 American Chemical Society, J. Am. Chem. Soc., Baar ja040021j Supporting Info Päge 1

INPORMATION

Kinetic Resolution of Chiral $\alpha$-Olefins Using Optically Active Ansa-Zirconocene

\title{
Polymerization Catalysts
}

Cliff R. Baar, Christopher J. Levy, Endy Y-J. Min, Lawrence M. Henling,

Michael W. Day, and John E. Bercaw*

Arnold and Mable Beckman Laboratories of Chemical Synthesis, California Institute of Technology, Pasadena

California 91125

Received xxxx xx, 2004

Supporting Information: X-ray data for $(S)-\mathbf{2},(S)-5,(S)-6$, and $(S)-7$ (CIF) (68 pages): This material is available free of charge via the Internet at http://pubs.acs.org. 
: (c) 2004 American Chemical Society, J. Am. Chem. Soc., Baar ja040021j Supporting Info Page 2

CIF FILE FOR ( $S)-2$

data_cj13

_audit_creation_method

_chemical_name_systematic.

SHELXL-97

;

?

;

_chemical_name_common

_chemical_melting_point

-chemical_formula_moiety

chemical_formula_sum

'C26 H42 Cl2 Si2 $\mathrm{Zr}$ '

_chemical_formula_weight

572.90

loop

atom_type_symbol

-atom_type_description

-atom_type_scat_dispersion_real

-atom_type_scat_dispersion_imag

_atom_type_scat_source

' $C$ ' ${ }^{\prime} \mathrm{C}$ ' $-0.00 \overline{3} 30.0016$

'International Tables Vol C Tables 4.2.6.8 and 6.1.1.4'

' H' 'H' $0.0000 \quad 0.0000$

'International Tables Vol C Tables 4.2.6.8 and 6.1.1.4'

'Si' 'Si' $0.0817 \quad 0.0704$

'International Tables Vol C Tables 4.2 .6 .8 and 6.1.1.4'

' $\mathrm{Cl}$ ' 'Cl' 0.14840 .1585

'International Tables Vol C Tables 4.2 .6 .8 and 6.1.1.4'

' $\mathrm{zr} \mathrm{C}^{\prime} \mathrm{zr}^{\prime} \quad-2.9673 \quad 0.5597$

'International Tables Vol C Tables 4.2 .6 .8 and 6.1.1.4'

symmetry_cell_setting

-symmetry_space_group_name_H-M

loop_

symmetry_equiv_pos_as_xyz

$x_{1}, z$

$'-x, y+1 / 2,-z '$

_cell_length_a

_cell_length_b

-cell_length_c

_cell_angle_alpha

_cell_angle_beta

_cell_angle_gamma
Monoclinic

P2(1)
$8.8568(8)$

$19.7932(18)$

$16.5826(15)$

90.00

$105.5220(10)$

90.00 
= (C) 2004 American Chemical Society, J. Am. Chem. Soc., Baar ja040021j Supporting Info Page 3

$=$

_cell_volume

cell_formula_units_z

cell_measurement_temperature

cell_measurement_reflns_used

_cell_measurement_theta_min

_cell_measurement_theta_max

exptl_crystal_description

-exptl_crystal_colour

-exptI_crystal_size_max

exptl_crystal_size_mid

_exptl_crystal_size_min

-exptl_crystal_density meas

-exptl_crystal_density_diffrn

-exptl_crystal_density_method

-exptl_crystal_F_000

expt1_absorpt_coefficient_mu

_exptl_absorpt_correction_type

exptl_absorpt_correction_T_min

_exptl_absorpt_correction_T_max

_exptI_absorpt_process_detaìls

_exptl_special_details

i

?

;

diffrn_ambient_temperature

diffrn_radiation wavelength

diffrn radiation type

diffrn_radiation_source

_diffrn_radiation_monochromator

_diffrn_measurement_device_type

_diffrn_measurement_method

_diffrn_detector_area_resol_mean

_diffrn_standards_number

diffrn_standards_interval_count

diffrn_standards_interval_time?

diffrn_standards_decay_o ?

diffrn_reflns_number

_diffrn_reflns_av_R_equivalents

_diffrn_reflns_av_sigmaI/netI

diffrn_reflns_limit_h_min

diffrn_reflns_limit_h_max

diffrn_reflns_limit_k_min

diffrn_reflns_limit_k_max

diffrn_reflns_limit_l_min

_diffrn_reflns_limit_l_max
$2801.0(4)$

4

$98(2)$

22851

2.386

26.309

Blade

Colorless

0.27

0.23

0.16

?

1.359

'not measured'

1200

0.681

none

0.8374

0.8988

?
$98(2)$

0.71073

MoK\a

'normal-focus sealed tube'

graphite

'Bruker SMART 1000'

' $\mathrm{W}$ scans at 6 If settings'

?

?

?

?

48755

0.0662

0.0616

$-11$

11

$-26$

25

$-21$

21 
= (C) 2004 American Chemical Society, J. Am. Chem. Soc., Baar ja040021j Supporting Info Page 4

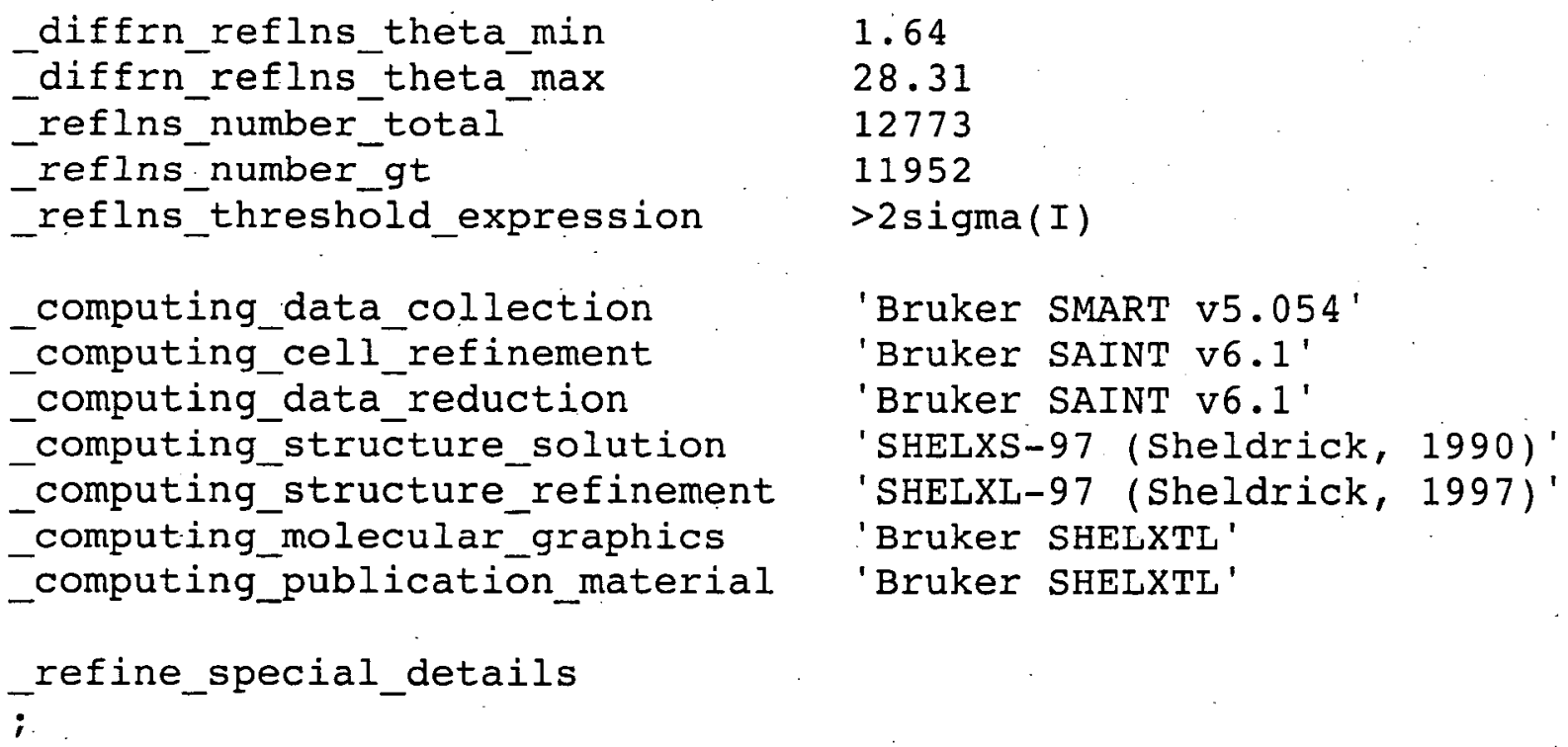

Refinement of $\mathrm{F}^{\wedge} 2^{\wedge}$ against ALI reflections. The weighted $\mathrm{R}$-factor $\mathrm{WR}$ and goodness of fit $S$ are based on $F^{\wedge} 2^{\wedge}$, conventional $R$-factors $R$ are based on $F$, with $F$ set to zero for negative $F^{\wedge} 2^{\wedge}$. The threshold expression of $\mathrm{F}^{\wedge} 2^{\wedge}>2 \mathrm{sigma}\left(\mathrm{F}^{\wedge} 2^{\wedge}\right)$ is used only for calculating R-factors(gt) etc. and is

not relevant to the choice of reflections for refinement. R-factors based

on $F^{\wedge} 2^{\wedge}$ are statistically about twice as large as those based on $F$, and R-

factors based on ALL data will be even larger. ;

refine_ls_structure_factor_coef Fsqd refine_ls_matrix type

-refine_ls_weighting_scheme

refine_ls_weighting_details

_atom_sites_solution_primary

-atom_sites_solution_secondary

-atom_sites_solution_hydrogens

refine_ls_hydrogen_treatment

-refine_ls_extinction_method

-refine_ls_extinction_coef

refine_ls_abs_structure_details

'Flack H D (1983), Acta Cryst. A39, 876-881'

_refine_ls_abs_structure_Flack $-0.01(4)$

_refine_ls_number_reflns

-refine_ls_number_parameters

12773

_refine_ls_number_restraints

-refine_ls_R_factor_all.

refine_ls_R_factor_gt

'Full matrix'

Sigma

' $\left.w=1 / \mathrm{s}^{\wedge} 2^{\wedge}\left(\mathrm{FO} \mathrm{O}^{\wedge}\right)^{\wedge}\right)^{\prime}$

'Patterson method'

'Difference Fourier map'

'Geometric positions'

Riding

none

?

refine_ls_wR_factor_ref

_refine_ls_wR_factor_gt

583

1

0.0375

0.0340

0.0582

0.0577 
= (C) 2004 American Chemical Society, J. Am. Chem. Soc., Baar ja040021j Supporting Info Page 5

$\begin{array}{ll}\text { refine_ls_goodness_of_fit_ref } & 1.212 \\ \text { _refine_ls_restrained_s_ali } & 1.212 \\ \text { refine_ls_shift/su_max } & 1.749 \\ \text { _refine_ls_shift/su_mean } & 0.041\end{array}$

loop

atom site label

_atom_site_type_symbol

-atom_site_fract_x

-atom_site_fract_y

-atom_site_fract_z

-atom_site_U_iso_or_equiv

-atom_site_adp_type

-atom_site_occupancy

-atom_site_symmetry_multiplicity

-atom_site_calc_flag

atom_site_refinement_flags

atom_site_disorder_assembly

atom_site_disorder_group

$\mathrm{z} \overline{\mathrm{r}} 1 \mathrm{~A}$ z $\overline{\mathrm{r}} 1.0 \overline{5} 251(6) \quad 0.27505(2) 0.50017(3) \quad 0.01205(12)$ Uani $11 \mathrm{~d}$. .

Cl1A Cl 1.05134(16) $0.21539(7) 0.37257(7) 0.0201(3)$ Uani $11 \mathrm{~d} .$.

Cl2A Cl 1.20981(17) $0.20049(6) 0.60753(7) 0.0189(3)$ Uani $11 \mathrm{~d} .$.

SilA Si $0.84613(18) 0.40269(7) \quad 0.41246(7) 0.0145(3)$ Uani $11 \mathrm{~d} .$.

Si2A Si $0.97863(18) 0.38214(7) \quad 0.62868(8) \quad 0.0137(3)$ Uani $11 \mathrm{~d} .$.

C1A C $0.7918(7) 0.3291(3) 0.4698(3) 0.0116(11)$ Uani $11 \mathrm{~d} . .$.

C2A C $0.8491(7) 0.3182(3) 0.5599(3) 0.0154(11)$ Uani $11 \mathrm{~d} .$.

C3A C $0.8460(6) 0.2466(2) 0.5741(3) 0.0097(11)$ Uani $11 d$. .

H3A H $0.87710 .2250 \quad 0.62710 .012$ Uiso 1 l calc $R$.

C4A C $0.7891(7) 0.2135(3) 0.4961(3) 0.0193(13)$ Uani $11 \mathrm{~d} . .$.

C5A C $0.7561(6) \quad 0.2641(3) \quad 0.4335(3) 0.0163(12)$ Uani $11 \mathrm{~d} .$.

H5A H $0.7156 \quad 0.2558 \quad 0.3753 \quad 0.020$ Uiso 11 calc R .

C6A C $1.0597(7) 0.3935(3) 0.4674(3) 0.0119(11)$ Uani $11 \mathrm{~d} . .$.

C7A C $1.1206(7) \quad 0.3858(2) 0.5609(2) 0.0122(11)$ Uani 11 d . .

C8A C $1.2734(7) 0.3595(3) .0 .5782(3) 0.0134(12)$ Uani 11 d . . .

C9A C $1.3079(7) 0.3468(3) 0.5008(3) 0.0140(12)$ Uani $11 d$. .

H9A.H $1.3999,0.32550 .49420 .017$ Uiso 11 calc R..

C10A C $1.1833(7) \quad 0.3710(3) 0.4351(3) 0.0151(12)$ Uani $11 \mathrm{~d}$. .

C11A C $0.7894(7) 0.3811(3) 0.2990(2) 0.0221(12)$ Uani $11 \mathrm{~d} .$.

H11A H $0.6749 \quad 0.3805,0.27810 .033$ Uiso 1 calc $R$. .

$\begin{array}{llllllllll}\text { H1 B H } & 0.8312 & 0.3365 & 0.2910 & 0.033 & \text { Uiso } 1 & 1 & \text { calc } R . .\end{array}$

$\begin{array}{llllllllll}\text { H1 C H } & 0.8322 & 0.4151 & 0.2681 & 0.033 & \text { Uiso } 1 \text { calc } R . .\end{array}$

C12A C $0.7543(7) \quad 0.4856(2) 0.4212(3) \quad 0.0233(12)$ Uani 11 d . .

H12A H $0.64140 .4796 \quad 0.41310 .035$ Uiso 11 calc $R$.

$\begin{array}{lllllllllll}\text { H12B H } & 0.7722 & 0.5164 & 0.3784 & 0.035 & \text { Uiso } 1 \text { calc } R . .\end{array}$

H12C H $0.8007 \quad 0.5048 \quad 0.4768 \quad 0.035$ Uiso 1 l calc $R$.

C13A C $0.8793(7) 0.4629(2) 0.6425(3) 0.0218(12)$ Uani $11 \mathrm{~d} .$.

$\begin{array}{llllllllll}\mathrm{H} 13 \mathrm{~A} & \mathrm{H} & 0.7901 & 0.4706 & 0.5937 & 0.033 & \text { Uiso } 1 & 1 & \text { calc } \mathrm{R} .\end{array}$

H13B H $0.9536 \quad 0.5004 \quad 0.64810 .033$ Uiso 1 i calc R.. 
: (c) 2004 American Chemical Society, J. Am. Chem. Soc., Baar ja040021j Supporting Info Page 6

$\mathrm{H} 13 \mathrm{C}$ H $0.8420 \quad 0.4601 \quad 0.6930 \quad 0.033$ Uiso $1 \quad 1$ calc $\mathrm{R}$.

C14A C $1.0623(7) \quad 0.3442(3) \quad 0.7344(3) \quad 0.0192(12)$ Uani 11 . . H14A H 0.97890 .33840 .76260 .029 Uiso 1 l calc $R$. .

H14B H $1.14330 .3741,0.76780 .029$ Uiso 11 calc $R$.

H $14 \mathrm{C}$ H $1.1085 \quad 0.3001 \quad 0.72840 .029$ Uiso 1 1 1 calc R . .

C15A C $0.7568(7) 0.1381(3) \quad 0.4789$ (3) $0.0143(12)$ Uani $11 \mathrm{~d}$. .

H15A H $0.7469 \quad 0.1320 \quad 0.4178 \quad 0.017$ Uiso 1 calc $R$.

C16A C $0.8975(6) \quad 0.0932(2) \quad 0.5249(3) \quad 0.0232(11)$ Uani 11 . . .

H16A H $0.9166 \quad 0.0990 \quad 0.58550 .035$ Uiso 1 l calc R .

H16B H 0.99110 .10670 .50810 .035 Uiso 1 calc $R$. .

$\mathrm{H} 16 \mathrm{C}$ H $0.8734 \quad 0.0457 \quad 0.5102 \quad 0.035$ Uiso 1.1 calc $\mathrm{R}$.

C17A C $0.5969(7) 0.1165(3) 0.4916(3) 0.0176(13)$ Uani $11 \mathrm{~d}$.

C18A C $0.4667(6) \quad 0.1654(3) \quad 0.4464(3) \quad 0.0274(12)$ Uani $11 d$.

H18A H 0.47450 .17330 .38930 .041 Uiso 1 1 calc R .

H18B H $0.4784 \quad 0.2084 \quad 0.4768 \quad 0.041$ Uiso 1 calc $R$. .

H18C H $0.3642 \quad 0.1458 \quad 0.4443 \quad 0.041$ Uiso 11 calc $R$. .

C19A C $0.5549(7) \quad 0.0446(2) \quad 0.4547(3) \quad 0.0299(13)$ Uani 11 e.. H19A H 0.44710 .03360 .45490 .045 Uiso 11 calc $R$. .

H19B H 0.62690 .01160 .48870 .045 Uiso 1 l calc $R$. .

H19C H 0.56420 .04340 .39710 .045 Uiso 1 calc R . .

C20A C $0.5998(6) 0.1147(2) 0.5856(3) \quad 0.0204(11)$ Uani 11 d . . H20A H $0.6287 \quad 0.1594 \quad 0.6104 \quad 0.031$ Uiso 11 calc R . .

H20B H $0.6768 \quad 0.08120 .6148 \quad 0.031$ Uiso 1 l calc $R$. •

H2OC H $0.4958 \quad 0.1023 \quad 0.5910 \quad 0.031$ Uiso 1 l calc $R$. .

C21A C $1.3870(7) \quad 0.3489$ (3) $0.6649(3) \quad 0.0162(12)$ Uani 11 e. . H21A H 1.33390 .31960 .69820 .019 Uiso 1 l calc R .

C22A C $1.4251(7) \quad 0.4166(3) \quad 0.7105(3) \quad 0.0224$ (13) Uani 1 l d . H22A H 1.48710 .40860 .76820 .034 . Uiso 11 calc R . .

H22B H 1.32750 .43970 .71090 .034 Uiso 1 calc $R$.

H22C H $1.48520 .44470 .6816 \quad 0.034$ Uiso 11 calc $\mathrm{R}$.

C23A C $1.5372(7) \quad 0.3133(3) \quad 0.6598(3) \quad 0.0225$ (14) Uani 1 l e. .

H23A H 1.59340 .34190 .62920 .034 Uiso 1 calc $\mathrm{R}$. .

H23B H $1.5107 \quad 0.27010 .6304 \quad 0.034$ Uiso 1 calc $\mathrm{R}$. .

H23C H $1.60390 .3050 \quad 0.71640 .034$ Uiso 1 calc R . .

C24A C $1.1881(7) \quad 0.3734(3) \quad 0.3424$ (3) $0.0155(12)$ Uani 11 . . H24A H $1.0898 \quad 0.3523 \quad 0.3074 \quad 0.019$ Uiso 1 calc $R$. .

C25A C $1.1921(7) \quad 0.4468(3) \quad 0.3156(3) \quad 0.0253(14)$ Uani $11 \mathrm{~d}$. .

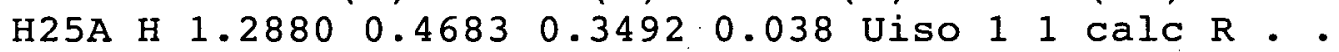

H25B H $1.1006 \quad 0.4706 \quad 0.3240 \quad 0.038$ Uiso I 1 calc R .

H25C H $1.1903 \quad 0.4486 \quad 0.2563 \quad 0.038$ Uiso 1 l calc $R$.

C26A C $1.3268(8) \quad 0.3352(3) \quad 0.3272$ (3) $0.0252(14)$ Uani 11 . .

H26A H 1.32110 .33620 .26740 .038 Uiso 1 l calc $\mathrm{R}$. .

H $26 \mathrm{~B} \mathrm{H} 1.32420 .28830 .3455 \quad 0.038$ Uiso 11 calc R . .

H26C H $1.4245 \quad 0.3566 \quad 0.35890 .038$ Uiso 1 l calc $R$. .

Zr1B Zr $1.05792(6) 0.72520(2) 0.99502(3) \quad 0.01247(13)$ Uani $11 d .$.

CI1B Cl $1.19746(17) \quad 0.79500(7) 1.11294(7) \quad 0.0199(3)$ Uani $11 d .$.

Cl2B Cl $1.09021(17) 0.79026(6) \quad 0.87634(7) \quad 0.0200(3)$ Uani $11 d$. .

SilB Si $0.96004(19) 0.60742(7) 1.10468(8) 0.0158(3)$ Uani 11 d . . 
Si2B Si $0.85050(18) \quad 0.60615(7) \quad 0.88658(8) 0.0142(3)$ Uani 11 d . . C1B C $0.8393(7) 0.6772(3) 1.0393(3) 0.0154(12)$ Uani $11 \mathrm{~d} .$. C2B C $0.7957(7) \quad 0.6767(3) \quad 0.9475(3) \quad 0.0132(11)$ Uani $11 d .$. Сзв C $0.7697(7) \quad 0.7464(2) \quad 0.9243(3) \quad 0.0157(12)$ Uani $11 d .$. H3B H $0.73810 .76190 .8680 \quad 0.019$ Uiso 11 calc $R$. C4B C $0.7965(6) \quad 0.7893(3) \quad 0.9946(3) \quad 0.0117(10)$ Uani $11 \mathrm{~d}$. . C5B C $0.8367(7) \quad 0.7439(3) \quad 1.0646(3) \quad 0.0174(13)$ Uani 11 d . . H5B H $0.85890 .7578 \quad 1.12140 .021$ Uiso 11 calc $\mathrm{R}$.

C6B C $1.1095(7) \quad 0.6091(3) 1.0419(3) 0.0131(11)$ Uani $11 \mathrm{~d}$. . C7B C $1.0659(7) \quad 0.6090(3) \quad 0.9495(3) \quad 0.0150(11)$ Uani $11 \mathrm{~d}$... C8B C $1.1934(6) \quad 0.6354(3) \quad 0.9235(3) \quad 0.0113(11)$ Uani $11 \mathrm{~d}$. . C9B C $1.3155(7) \quad 0.6524(3) \quad 0.9967(3) 0.0151(12)$ Uani 11 . . . H9B H 1.41320 .67210 .99660 .018 Uiso 1 1 calc R : . C10B C $1.2687(6) 0.6356(3) 1.0686(3) \quad 0.0127(12)$ Uani $11 \mathrm{~d}$... C11B C $1.0306(7) \quad 0.6400(3) 1.2137(3) \quad 0.0195(12)$ Uani $11 d .$. H11D H $0.9410 \quad 0.64721 .23680 .029$ Uiso 1 l calc $R$. . H11E H $1.0857 \quad 0.68291 .21340 .029$ Uiso 11 calc $R$. . H11F H $1.1023 \quad 0.6071 \quad 1.24820 .029$ Uiso 1 l calc R . . C12B C $0.8602(7) \quad 0.5249(2) \quad 1.1112(3) \quad 0.0208(12)$ Uani $11 d$. H12D H 0.82470 .52391 .16230 .031 Uiso 1 l calc $R$. . H12E H $0.93390 .4877 \quad 1.11240 .031$ Uiso 1 l calc $R$. . H $12 \mathrm{~F}$ H 0.76990 .51991 .06230 .031 Uiso 1 l calc $R$. . C13B C $0.7531(8) \quad 0.5221(3) \quad 0.8876(3) \quad 0.0294(14)$ Uani 11 . . H13D H $0.6390 \quad 0.5280 \quad 0.8706 \quad 0.044$ Uiso 11 calc $R$. . H13E H. 0.78510 .50310 .94420 .044 Uiso 11 calc R . . H13F H. $0.78410 .49140 .8486 \quad 0.044$ Uiso 11 calc $\mathrm{R}$. . C14B C $0.8056(7) \quad 0.6344(3) \quad 0.7761(3) \quad 0.0216(12)$ Uani $11 d .$. H14D H 0.86110 .67680 .77290 .032 Uiso 1 calc $\mathrm{R}$. . H14E H 0.69250 .64170 .75450 .032 Uiso 1 l calc R . .

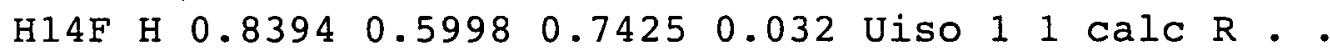
C15B C $0.7806(6) \quad 0.8653(3) \quad 1.0010(3) 0.0154(11)$ Uani 11 . . H15B H $0.83310 .8770 \quad 1.0606 \quad 0.018$ Uiso 1 I calc $\mathrm{R}$. . C16B C $0.8699(6) \quad 0.9041(2) \quad 0.9483(3) \quad 0.0254(12)$ Uani $11 d .$. H16D H 0.98270 .89630 .97080 .038 Uiso 11 calc R . . H16E H 0.84770 .95250 .95010 .038 Uiso 1 l calc $R$. . H $16 \mathrm{~F}$ H 0.83650 .88820 .89020 .038 Uiso 1 calc $\mathrm{R}$. . C17B C $0.6059(7) \quad 0.8869(3) \quad 0.9861(3) \quad 0.0177(13)$ Uani 11 d . . C18B C $0.5225(6) 0.8418(2) 1.0360(3) \quad 0.0242(12)$ Uani 11 e . $\mathrm{H} 18 \mathrm{D} \mathrm{H} 0.42010 .86131 .03450 .036$ Uiso 1 calc $\mathrm{R}$. . H18E H 0.58610 .83891 .09420 .036 Uiso 1 l calc R . . $\mathrm{H} 18 \mathrm{~F}$ H $0.5080 \quad 0.79661 .01130 .036$ Uiso 1.1 calc $\mathrm{R}$. . C19B C $0.6010(6) 0.9604(2) 1.0160(3) \quad 0.0252(12) \operatorname{Uani} 11 \mathrm{~d}$. . H19D H 0.49180 .97551 .00390 .038 Uiso 11 Calc R . . H19E H $0.6584 \quad 0.98960 .98670 .038$ Uiso 1 l calc $R .$. H19F H $0.6496 \quad 0.9628 \quad 1.07640 .038$ Uiso 1 l calc R . . C20B C $0.5148(6) \quad 0.8820(2) \quad 0.8919(3) \quad 0.0245(11)$ Uani 11 d . . H2OD H $0.5176 \quad 0.83520 .8728 \quad 0.037$. Uiso 1 l calc R . . $\mathrm{H} 20 \mathrm{E}$ H $0.5638 \quad 0.91170 .8590 \quad 0.037$ Uiso 1 calc R . 
H $20 \mathrm{~F} \mathrm{H} 0.4058 \quad 0.8958 \quad 0.8848 \quad 0.037$ Uiso 11 calc $R$.

C21B C $1.2068(7) \quad 0.6399(3) \quad 0.8344(3) \quad 0.0154(11)$ Uani $11 \mathrm{~d} .$.

H21B H $1.11540 .66640 .8008 \quad 0.019$ Uiso 1 calc $\mathrm{R}$.

C22B C $1.1999(9) \quad 0.5680(2) \quad 0.7956(3) \quad 0.0241(14)$ Uani 11 . .

H22D H 1.29530 .54310 .8233 .0 .036 Uiso 1 calc R . .

H22E H 1.19180 .57180 .73570 .036 Uiso 1 calc $R$. .

$\mathrm{H} 22 \mathrm{~F} \quad \mathrm{H} \quad 1.1082 \quad 0.54390 .8035 \quad 0.036$ Uiso 1 l calc $\mathrm{R}$. .

C23B C $1.3554(7) \quad 0.6750(3) \quad 0.8286(3) \quad 0.0222(14)$ Uani 11 . .

H23D H 1.34730 .72350 .83890 .033 Uiso 1.1 calc $R$ • •

H23E H $1.36990 .66820 .7726 \quad 0.033$ Uiso 1 l calc $R$. .

$\begin{array}{llllllllll}\mathrm{H} 23 \mathrm{~F} & \mathrm{H} & 1.4451 & 0.6561 & 0.8706 & 0.033 & \text { Uiso } 1 & 1 & \text { calc } \mathrm{R} \text {. }\end{array}$

C24B C $1.3726(7) \quad 0.6390(3) 1.1575(3) 0.0167(12)$ Uani $11 d$. 1 .

$\mathrm{H} 24 \mathrm{~B}$ H $1.3128 \quad 0.6624 \quad 1.19270 .020$ Uiso 11 calc $\mathrm{R}$. .

C25B C $1.4110(8) \quad 0.5668(2) 1.1920(3) 0.0260(14)$ Uani $11 d$.

H25D H 1.46630 .54241 .15710 .039 Uiso 1 l calc $R$. •

H25E H $1.31350 .5431 \quad 1.19120 .039$ Uiso 1 1 calc $R$. .

$\mathrm{H} 25 \mathrm{~F}$ H $1.47750 .5691 \quad 1.2496 \quad 0.039$ Uiso 1 calc $\mathrm{R}$. .

C26B C $1.5242(8) 0.6779$ (3) $1.1653(3) \quad 0.0294(16)$ Uani 11 d .

H26D H 1.58520 .67881 .2240 .0 .044 Uiso 11 calc $R$.

H26E H $1.4996 \quad 0.72421 .1452 \quad 0.044$ Uiso 11 calc $R$ : .

H26F H $1.5853 \quad 0.6556 \quad 1.13160 .044$ Uiso 1 l calc R .

loop

_atom_site_aniso_label

-atom_site_aniso_U_11

-atom_site_aniso_U_22

-atom_site_aniso_U_33

-atom_site_aniso_U_23

atom_site_aniso_U_13

atom_site_aniso_U_12

$\mathrm{z} \overline{\mathrm{r}} 1 \mathrm{~A} \quad 0.0150 \overline{(3)} 0 . \overline{0} 1 \overline{0} 9(3) \quad 0.0099(2)-0.00010(18) \quad 0.0028(2) \quad 0.0001(2)$

Cl1A $0.0230(7) \quad 0.0210(7) \quad 0.0170(5)-0.0064(5) \quad 0.0063(5)-0.0016(6)$

Cl2A $0.0198(7) \quad 0.0163(7) \quad 0.0180(6) \quad 0.0032(5) \quad 0.0003(5) \quad 0.0006(6)$

SilA $0.0165(8) \quad 0.0143(7) \quad 0.0111(6)-0.0001(5) \quad 0.0013(6) \quad 0.0005(6)$

$\operatorname{si2A} 0.0165(8) \quad 0.0121(7) \quad 0.0131(6)-0.0015(5) \quad 0.0051(6)-0.0015(6)$

C1A $0.010(3) \quad 0.011(3) \quad 0.016(2) \quad 0.0050(19) \quad 0.007(2) \quad 0.000(2)$

$\mathrm{C} 2 \mathrm{~A} \quad 0.009(3) \quad 0.023(3) \quad 0.012(2)-0.0033(19)-0.002(2) \quad 0.001(2)$

C3A $0.0 .10(3) \quad 0.003(2) \quad 0.018(2) \quad 0.0010(17) \quad 0.006(2) \quad-0.0019(19)$

$\begin{array}{lllllll}C 4 A & 0.016(3) & 0.020(3) & 0.021(3) & 0.001(2) & 0.003(2) & 0.002(3)\end{array}$

C5A $0.014(3) \quad 0.022(3) \quad 0.015(2)-0.002(2) \quad 0.006(2)-0.004(2)$

$\begin{array}{lllllll}\mathrm{C} 6 \mathrm{~A} & 0.019(3) & 0.007(2) & 0.011(2) & 0.0003(17) & 0.007(2) & 0.001(2)\end{array}$

C7A $0.026(3) \quad 0.002(2) \quad 0.0062(19)-0.0018(16)-0.001(2)-0.003(2)$

$\mathrm{C} 8 \mathrm{~A} \quad 0.021(3) \cdot 0.008(3) \quad 0.013(2)-0.002(2) \quad 0.007(2)-0.005(2)$

C9A $0.017(3) \quad 0.010(3) \quad 0.020(3) \quad 0.007(2) \quad 0.013(2) 0.006(2)$

$\mathrm{C} 10 \mathrm{~A} \quad 0.028(3) \quad 0.008(3) \quad 0.011(2)-0.0019(19) 0.007(2)-0.002(2)$

CI1A $0.029(3) \quad 0.024(3) \quad 0.011(2)-0.0018(19)-0.001(2)-0.008(2)$

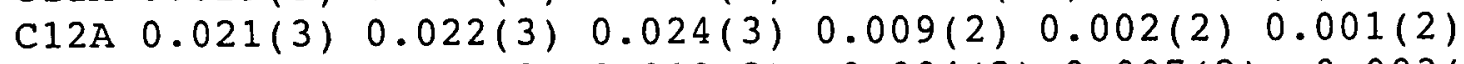

$\mathrm{C} 13 \mathrm{~A} \quad 0.028(3) \quad 0.018(3) \quad 0.019(2)-0.004(2) \quad 0.007(2)-0.003(2)$ 
(C) 2004 American Chemical Society, J. Am. Chem. Soc., Baar ja040021j Supporting Info Page 9

\begin{tabular}{|c|c|c|c|c|}
\hline C14A & $0.021(3)$ & $0.027(3)$ & $0.010(2)$ & $0.005(2)-0.004(2)$ \\
\hline C15A & $0.024(3)$ & $0.010(3)$ & $0.008(2)$ & $-0.0013(17)$ \\
\hline C16A & $18(3)$ & $11(2)$ & $.039(3)$ & $-0.0037(19) 0.005(2)-0.001(2)$ \\
\hline $7 \mathrm{~A}$ & $017(3)$ & $0.014(3)$ & $0.020(3)$ & $-0.002(2) \quad 0.002(2) \quad 0.003(2)$ \\
\hline $18 \mathrm{~A}$ & $16(3)$ & $0.032(3)$ & $0.029(3)$ & $0.000(2)-0.002(2)$ \\
\hline $19 A$ & $31(4)$ & $23(3)$ & $41(3)$ & $-0.009(2) \quad 0.017(3)-0.010(3)$ \\
\hline $20 A$ & $022(3)$ & $14(2)$ & $24(2)$ & $0.0039(19) 0.004(2) 0.000(2)$ \\
\hline $21 \mathrm{~A}$ & $0.013(3)$ & $0.018(3)$ & $0.012(2)$ & $-0.0034(19)-0.007(2) 0.003(2)$ \\
\hline C22A & $0.024(3)$ & $0.028(3)$ & $0.014(2)$ & $0.000(2) 0.001(2)-0.003(3)$ \\
\hline $23 \mathrm{~A}$ & $0.013(3)$ & $0.030(3)$ & $0.020(3)$ & $0.003(2)-0.003(2) 0.006(3)$ \\
\hline $24 \mathrm{~A}$ & $18(3)$ & $0.019(3)$ & $0.010(2)$ & $-0.0043(19) 0.003(2)-0.001(2)$ \\
\hline$C 25 A$ & $0.039(4)$ & $0.023(3)$ & $0.014(2)$ & $-0.003(2) \quad 0.008(2)-0.001(3)$ \\
\hline $26 \mathrm{~A}$ & $0.035(4)$ & $0.027(3)$ & $0.017(2)$ & $-0.001(2) 0.012(2)-0.005(3)$ \\
\hline $1 \mathrm{~B}$ & $141(3)$ & & 3) 0.0100 & (2) $0.0002(2) \quad 0.0026(2)-0.0010(2)$ \\
\hline $11 \mathrm{~B}$ & $80(7)$ & & 0174 & $-0.0058(5) \quad 0.0024(5)-0.0029(6)$ \\
\hline $12 \mathrm{~B}$ & $0.0273(8)$ & $0.0169(7$ & 0.0179 & $0.0048(5) \quad 0.0098(5)-0.0001(5)$ \\
\hline ilB & $0.0191(8)$ & $0.0155(7$ & 0.0130 & $0.0011(5)$ \\
\hline i2B & $0153(8)$ & & 0.0107( & $0.0010(5) 0.0011(6)-0.0026(6)$ \\
\hline $1 \mathrm{BC}$ & $0.014(3)$ & $0.013(3)$ & $0.020(3) 0$ & $0.000(2) 0.006(2)-0.002(2)$ \\
\hline $2 B$ & $.012(3)$ & $0.019(3)$ & $0.009(2)$ & $0.0018(19) 0.003(2)-0.003(2)$ \\
\hline $3 B$ & $(3)$ & $0.017(2)$ & $0.012(2)$ & $0.0050(18) .0 .002(2) .0 .001(2)$ \\
\hline $4 \mathrm{~B}$ & $8(3)$ & $0.010(3)$ & $0.017(2)$ & $-0.0008(19) \quad 0.002(2) 0.000(2)$ \\
\hline $5 B$ & $0.014(3)$ & $0.027(3)$ & $0.011(2)$ & $-0.004(2) \quad 0.004(2)-0.005(3)$ \\
\hline $6 \mathrm{~B}$ & $0(3)$ & $0.009(2)$ & $0.020(2)$ & $-0.004(2) \quad 0.004(2)-0.001(2)$ \\
\hline $7 \mathrm{~B}$ & $0.020(3)$ & $0.013(3)$ & $0.009(2)$ & $0.0005(19) 0.000(2) 0.002(2)$ \\
\hline $28 \mathrm{~B}$ & $0.011(3)$ & $0.007(2)$ & $0.015(2)$ & $0.0014(18) 0.001(2)-0.001(2)$ \\
\hline C9B C & $0.013(3)$ & $0.017(3) 0$ & $0.017(3)$ & $0.005(2) .0 .006(3) 0.006(2)$ \\
\hline $210 \mathrm{~B}$ & $0.011(3)$ & $0.010(3)$ & $0.014(2)$ & $-0.003(2)-0.001(2) 0.005(2)$ \\
\hline $\mathrm{C} 11 \mathrm{~B}$ & $0.022(3)$ & $0.024(3)$ & $0.015(2)$ & $-0.001(2) 0.008(2) 0.008(3)$ \\
\hline C12B & $0.023(3)$ & $0.021(3)$ & $0.018(2)$ & $0.001(2) 0.004(2)-0.002(2)$ \\
\hline $13 \mathrm{~B}$ & $0.034(4)$ & $0.032(3)$ & $0.023(3)$ & $-0.010(2) \quad 0.009(3)-0.014(3)$ \\
\hline C14B & $0.024(3)$ & $0.026(3)$ & $0.011(2)$ & $0.002(2) \cdot-0.001(2)$ \\
\hline C15B & $0.018(3)$ & $0.015(3)$ & $0.013(2)$ & $-0.001(2) 0.003(2)$ \\
\hline C16B & $0.024(3)$ & $0.020(3)$ & $0.037(3)$ & $-0.006(2)$ \\
\hline C17B & $0.023(3)$ & $0.016(3)$ & $0.016(2)$ & $-0.001(2) \quad 0.009(2)-0.002(2)$ \\
\hline C18B & $0.025(3)$ & $0.024(3)$ & $0.024(2)$ & $0.003(2) 0.008(2) 0.006(2)$ \\
\hline C19B & $0.027(3)$ & $0.027(3)$ & $0.021(3)$ & $-0.0044(19) \quad 0.004(2) \quad 0.007(2)$ \\
\hline $\mathrm{C} 20 \mathrm{~B}$ & $0.020(3)$ & $0.025(3)$ & $0.026(2)$ & $-0.004(2) 0.001(2) 0.006(2)$ \\
\hline $\mathrm{C} 21 \mathrm{~B}$ & $0.017(3)$ & $0.014(3)$ & $0.014(2)$ & $0.0024(18) 0.003(2) 0.002(2)$ \\
\hline C22B & $0.045(4)$ & $0.016(3)$ & $0.011(2)$ & $0.0038(18) 0.008(2) 0.009(3)$ \\
\hline C23B & $0.018(3)$ & $0.032(3)$ & $0.020(3)$ & $0.005(2) 0.011(2)-0.001(3)$ \\
\hline $\mathrm{C} 24 \mathrm{~B}$ & $0.017(3)$ & $0.016(3)$ & $0.018(2)$ & $-0.0039(19) \quad 0.007(2) \quad 0.003(2)$ \\
\hline & $0.030(4)$ & $0.029(3)$ & $0.016(2)$ & $0.005(2) \quad 0.002(2) 0.000(3)$ \\
\hline$C 26 B$ & $0.032(4)$ & $0.035(4)$ & $0.018(3)$ & $0.002(2) 0.002(3) 0.005(3)$ \\
\hline
\end{tabular}

_geom_special_details ;

All esds (except the esd in the dihedral angle between two 1.5 . planes) 
(C) 2004 American Chemical Society, J. Am. Chem. Soc., Baar ja040021j Supporting Info Page 10

are estimated using the full covariance matrix. The cell esds are taken into account individually in the estimation of esds in distances, angles and torsion angles; correlations between esds in cell parameters are only used when they are defined by crystal symmetry. An approximate

(isotropic)

treatment of cell esds is used for estimating esds involving 1.s. planes. ;

loop

_geom_bond_atom_site_label_1

-geom_bond_atom_site_label_2

geom_bond_distance

geom_bond_site_symmetry_2

geombond_publ_flag

$\overline{\mathrm{Z}} \overline{\mathrm{IA}} \operatorname{Cent}(1 \overline{\mathrm{A}}) 2 . \overline{2} 11$. yes

$\mathrm{Zr} 1 \mathrm{~A}$ Cent (2A) 2.241 . yes

$\mathrm{Zr} 1 \mathrm{~A} P \ln (1 \mathrm{~A}) 2.2013(25)$. yes

$\mathrm{Zr} 1 \mathrm{~A} P \ln (2 \mathrm{~A}) 2.2151(27)$. yes

Zr1A C6A 2.412(5).?

Zr1A C7A 2.420(5) : ?

ZrlA Cl1A 2.4209(13) . yes

Zr1A Cl2A 2.4415(14) . yes

Zr1A C2A 2.431(6).?

$\operatorname{Zr1A}$ C1A $2.471(6)$ ?

Zr1A C3A 2.523(5).?

Zr1A C5A 2.570(6) . ?

$\operatorname{Zr} 1 \mathrm{~A}$ C10A 2.605(5). ?

Zr1A C4A 2.617(6) .?

Zr1A C8A 2.635(5).?

Zr1A C9A $2.669(6)$. ?

Si1A C12A 1.855(5). ?

Si1A C1A $1.872(5)$ ?

SilA C1IA 1.863(4) . ?

Si1A C6A $1.876(6)$. ?

Si2A C13A $1.868(5) \cdot$ ?

Si2A C14A $1.869(4)$. ?

Si2A C2A $1.876(6)$. ?

Si2A C7A 1.898(6) ?

C1A C5A $1.418(7)$ ?

C1A C2A 1.458(6) ?

C2A C3A $1.439(7)$. ?

C3A C4A $1.416(6) \cdot$ ?

C4A C5A 1.416(7) . ?

C4A C15A $1.532(8)$. ?

C6A C10A 1.413(7).?

C6A C7A 1.508(5) . ?

C7A C8A 1.406(8) . ?

C8A C9A $1.420(6)$ ? 


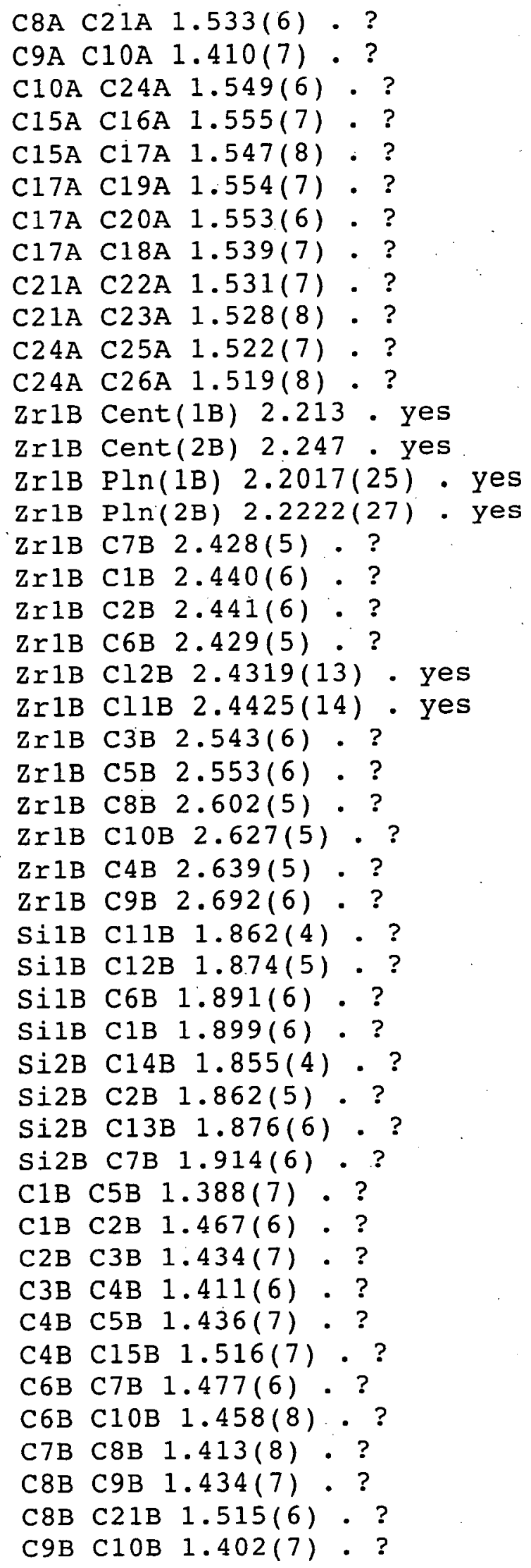


(C) 2004 American Chemical Society, J. Am. Chem. Soc., Baar ja040021j Supporting Info Page 12

$\begin{array}{lllll}\mathrm{C} 10 \mathrm{~B} & \mathrm{C} 24 \mathrm{~B} & 1.515(7) & . & ? \\ \mathrm{C} 15 \mathrm{~B} & \mathrm{C} 17 \mathrm{~B} & 1.560(8) & . & ? \\ \mathrm{C} 15 \mathrm{~B} & \mathrm{C} 16 \mathrm{~B} & 1.533(7) & . & ? \\ \mathrm{C} 17 \mathrm{~B} & \mathrm{C} 18 \mathrm{~B} & 1.535(7) & . & ? \\ \mathrm{C} 17 \mathrm{~B} & \mathrm{C} 20 \mathrm{~B} & 1.556(6) & . & ? \\ \mathrm{C} 17 \mathrm{~B} & \mathrm{C} 19 \mathrm{~B} & 1.541(7) & . & ? \\ \mathrm{C} 21 \mathrm{~B} & \mathrm{C} 23 \mathrm{~B} & 1.513(7) & . & ? \\ \mathrm{C} 21 \mathrm{~B} & \mathrm{C} 22 \mathrm{~B} & 1.556(6) & . & ? \\ \mathrm{C} 24 \mathrm{~B} & \mathrm{C} 26 \mathrm{~B} & 1.522(8) & . & ? \\ \mathrm{C} 24 \mathrm{~B} & \mathrm{C} 25 \mathrm{~B} & 1.544(7) & .\end{array}$

loop _geom_angle_atom_site_label_1 -geom_angle_atom_site_label_2 -geom_angle_atom_site_label_3 _geom_angle _geom_angle_site_symmetry_1 -geom_angle_site_symmetry_3 -geom_angle_publ_flag

Cent $(1 \bar{A}) \operatorname{Zr} 1 \bar{A} \operatorname{Cent}(2 A) 121.7$. . yes $P \ln (1 A) \operatorname{Zr} 1 A$ Pln(2A) 107.95(25) - . yes C6A ZrIA C7A $36.37(13)$. . ? C6A ZrIA Cl1A 105.75(11) . ? C7A Zr1A CI1A 138.42(12) . . ? C6A Zr1A C12A 135.06(13) . . ? C7A Zr1A Cl2A 102.34(11) . ? CI1A Zr1A Cl2A 102.15(5) . . yes C6A Zr1A C2A 79.46(18) . . ? C7A Zr1A C2A 68.99(19) . . ? CI1A ZIIA C2A 134.19(13). . ?

C12A Zr1A C2A 104.83(12)..? C6A Zr1A C1A 67.23(18)..? C7A Zr1A C1A 78.94(19) •. ? C11A Zr1A C1A 104.36(12)..? C12A 2r1A C1A 136.87(12) . . ? C2A Zr1A C1A 34.61(15) . ? C6A Zr1A C3A 113.13(17) . . ? C7A 2 r1A C3A 97.60(17) . ? Cl1A Zr1A C3A 118.79(11) . . ? C12A Zr1A C3A 81.79(12) ..? C2A Zr1A C3A 33.70(16) . ? C1A Zr1A C3A 55.68(16) ..? C6A Zr1A C5A 94.03(18)..? C7A Zr1A C5A 111.54(18) . . ? C11A Zr1A C5A 79.53(11)..? C12A 2r1A C5A 125.49(12) . ? C2A Zr1A C5A 54.67(16) . ? C1A Zr1A C5A 32.61(16) . ? 
(C) 2004 American Chemical Society, J. Am. Chem. Soc., Baar ja040021j Supporting Info Page 13

C3A Zr1A C5A 53.15(17) . ?

C6A 2 IIA C10A $32.44(17)$. . ?

C7A $\operatorname{Zr} 1 \mathrm{~A}$ C10A $54.77(16)$. ?

CIIA ZIIA C10A 83.80(11) . : ?

CI2A $\operatorname{Zr} 1 \mathrm{~A}$ C1OA $120.84(14) \cdot$. ?

C2A ZrIA C10A $111.88(18)$. ?

C1A Zr1A C10A 95.46(18) . ?

C3A Zr1A C10A $145.57(17)$. . ?

C5A Zr1A C10A 113.55(17) . ?

C6A Zr1A C4A 121.60(19) . . ?

C7A Zr1A C4A 123.60(19) . . ?

Cl1A Zr1A C4A 87.44(12) . . ?

C12A Zr1A C4A 93.90(13) . . ?

C2A Zr1A C4A 54.64(18) ..?

C1A Zr1A C4A 54.43(17) ..?

C3A Zr1A C4A 31.91(14) . . ?

C5A Zr1A C4A 31.67(15) . . ?

C10A ZIIA C4A 145.22(17) ..?

C6A Zr1A C8A 55.45(17) - .?

C7A Zr1A C8A 31.94(18) . . ?

CI1A ZIIA C8A 124.59(12) . ?

C12A ZrIA C8A 79.73(12) . : ?

C2A Zr1A C8A 96.37(18) : ?

C1A Zr1A C8A $110.86(18)$...?

C3A Zr1A C8A $116.28(15)$. ?

C5A Zr1A C8A 143.44(17) . . ?

C10A Zr1A C8A 52.15(16) . ?

C4A Zr1A C8A 147.97(15) . ?

C6A Zr1A C9A 53.84(18) •.?

C7A Zr1A C9A 53.13(18) .. ?

C11A Zr1A C9A 93.91(11) ..?

C12A Zr1A C9A 90.08(12) ..?

C2A Zr1A C9A 122.08(18) .. ?

C1A Zr1A C9A 121.02(18) . ?

C3A $\operatorname{Zr1A}$ C9A $147.25(15)$. ?

C5A Zr1A C9A 144.43(16) .. ?

C10A Zr1A C9A 30.99(16) . . ?

C4A Zr1A C9A 175.44(19) . . ?

C8A Zr1A C9A 31.04(14) . ?

C12A SilA C1A $118.1(2) \cdot$. ?

C12A SilA C11A 106.0(2) . . ?

C1A SIIA C11A 107.4(2) : ?

C12A SilA C6A 117.0(2) . ?

C1A SilA C6A 92.3(2) . . ?

C11A SilA C6A 115.6(2) . . ?

C12A SilA $\operatorname{Zr} 1 \mathrm{~A} 149.86(16)$. ?

C1A SilA Zr1A 49.70(18) . . ?

C1IA SilA Zr1A 104.08(17) . ? 
C6A SilA Zr1A $47.89(16)$. ?

C13A Si2A C14A 108.1(2) . . ?

C13A Si2A C2A $114.8(3)$. ?

C14A.SI2A C2A $108.9(2)$. . ?

C13A SI2A C7A $116.7(2) \cdot$. ?

C14A SI2A C7A $114.3(2)$. ?

C2A Si2A C7A $93.4(2)$. ?

C13A Si2A Zr1A $146.79(15)$. . ?

C14A SI2A Zr1A $104.98(16)$. : ?

C2A Si2A $\operatorname{Zr} 1 \mathrm{~A} 49.30(18)$. ?

C7A Si2A Zr1A 49.02(14) ..?

C5A C1A C2A 106.1(4) . ?

C5A C1A SIIA 123.2(4) •.?

C2A C1A SIIA 124.7(4) •.?

C5A C1A Zr1A 77.6(3) . ?

C2A C1A Zr1A 71.2(3) . ?

SIIA C1A ZRIA 95.0(2) . ?

C3A C2A C1A 107.2(4) . ?

C3A C2A Si2A 126.9(4) •. ?

C1A C2A Si2A 120.8(4) . . ?

C3A C2A $\operatorname{Zr1A} 76.6(3)$. ?

C1A C2A Zr1A 74.2(3) : ?

Si2A C2A $\operatorname{Zr} 1 A$ 94.9(2) . ?

C4A C3A C2A 108.9(4) ..?

C4A C3A Zr1A 77.7(3) . . ?

C2A C3A Zr1A 69.7(3) . : ?

C3A C4A C5A 107.1(5). ?

C3A C4A C15A 128.6(4) . ?

C5A C4A C15A 124.1(4)..?

C3A C4A Zr1A 70.4(3) . ?

C5A C4A Zr1A 72.4(3). ?

C15A C4A ZrIA 125.4(4) . ?

C4A C5A C1A $110.6(4)$. ?

C4A C5A $\operatorname{2r} 1 A 76.0(3) \cdot$ ?

C1A C5A Zr1A 69.8(3)..?

C10A C6A C7A 104.9(4) $\cdot$ ?

C10A C6A SilA 129.0(4) :.?

C7A C6A Si1A 123.1(4) •. ?

C10A C6A Zr1A 81.3(3)..'?

C7A C6A Zr1A 72.1(2) . ?

Si1A C6A Zr1A 96.9(2) •.?

C8A C7A C6A 107.9(4) . ?

C8A C7A Si2A 128.3(3) . . ?

C6A C7A Si2A $120.1(4)$. ?

C8A C7A 2r1A 82.5(3) ..?

C6A C7A Zr1A 71.5(2) ..?

Si2A C7A $\operatorname{Zr} 1 A$ 94.7(2) . ?

C7A C8A C9A 108.0(4) ..? 
(C) 2004 American Chemical Society, J. Am. Chem. Soc., Baar ja040021j Supporting Info Page 15

C7A C8A C21A 126.6(4). ?

C9A C8A C21A $125.3(5)$. ?

C7A C8A Zr1A 65.6(3)..?

C9A C8A Zr1A 75.8(3) . ?

C2IA C8A ZRIA $126.0(3)$. ?

C8A. C9A C10A 109.0(5) ..?

C8A C9A Zr1A 73.2(3) . ?

C10A C9A Zr1A 72.0(3) . . ?

C6A C10A C9A $109.9(4)$. ?

C6A C10A C24A $126.6(5)$. ?

C9A C10A C24A $123.5(5)$. ? ?

C6A C10A Zr1A 66.2(3) •.?

C9A CIOA $\operatorname{ZrIA~} 77.0(3)$. ?

C24A C10A ZrIA 124.5(3) • ?

C4A C15A C16A 112.3(4) . ?

C4A C15A C17A $112.1(5)$. ?

C16A C15A C17A 114.7(4) . ?

C19A C17A C20A 108.2(4) •.?

C19A C17A C18A 108:4(4) •.?

C20A C17A C18A 108.2(4) •.?

C19A C17A C15A 109.3(4) •.?

C20A C17A C15A 111.9(4) •.?

C18A C17A C15A $110.7(5)$. ?

C22A C21A C8A $110.2(4) . \cdot ?$

C22A C21A C23A $110.5(5)$. ?

C8A C21A C23A $111.9(4)$. ?

C25A C24A C26A 110.0(5) . ?

C25A C24A C10A 109.1(4)..?

C26A C24A C10A $112.8(4)$. ?

Cent(1B) $\operatorname{Zr1B}$ Cent(2B) 122.2 . yes

$\mathrm{P} \ln (1 \mathrm{~B}) \mathrm{Zr} 1 \mathrm{~B} \operatorname{Pln}(2 \mathrm{~B}) 107.81(23) \cdot$. Yes

C7B Zr1B C1B $79.39(19)$. . ?

$\mathrm{C} 7 \mathrm{~B} \quad \mathrm{Zr} 1 \mathrm{~B}$ C2B $68.4(2)$. . ?

C1B Zr1B C2B $34.99(14)$. ?

C7B $\operatorname{Zr1B}$ C6B $35.40(14)$. . ?

C1B Zr1B C6B $68.39(19)$. ?

C2B Zr1B C6B 79.20(18) •.?

C7B Zr1B Cl2B 103.33(11) • ?

C1B Zr1B C12B 136.43(13) •. ?

C2B $\operatorname{Zr} 1 \mathrm{~B}$ Cl2B $104.76(11) \cdot . ?$

C6B Zr1B Cl2B 134.98(12) • ?

C7B Zr1B Cl1B $137.24(13) \cdot$ ?

C1B Zr1B Cl1B $104.58(12)$. ?

C2B Zr1B Cl1B 135.63(12) . ?

C6B Zr1B Cl1B 105.50(12) . ?

C12B Zr1B CI1B $101.80(5)$. . yes

C7B $\operatorname{Zr1B}$ C3B 97.00(18) . ?

CIB ZrIB C3B $54.80(17)$. ? 
(C) 2004 American Chemical Society, J. Am. Chem. Soc., Baar ja040021j Supporting Info Page 16

U

C2B 2 r1B C3B $33.37(16) \div$. ?

C6B 2r1B C3B $112.54(18)$. ?

С 2B $\operatorname{Zr} 1 \mathrm{~B}$ С3в $81.97(11)$. ?

Cl1B ZI1B C3B $120.43(12)$ : : ?

C7B $\operatorname{Zr} 1 \mathrm{~B}$ C5B $111.56(19)$. ?

C1B 2 r1B C5B $32.17(17)$. ?

$\mathrm{C} 2 \mathrm{~B}$ Z $11 \mathrm{~B}$ C5B $54.82(17)$. ?

C6B $\operatorname{Zr} 1 \mathrm{~B}$ C5B $95.38(18)$. . ?

C12B Zr1B C5B 124.04(13) . ?

Cl1B $\operatorname{Zr} 1 \mathrm{~B}$ C5B $80.87(12)$. ?

C3B 2r1B C5B 52.23(17) . ?

C7B Zr1B C8B 32.39(18) . ?

C1B Zr1B C8B 111.77(17) . . ?

C2B Zr1B C8B 95.69(17)..?

C6B $\operatorname{Zr} 1 \mathrm{~B}$ C8B 55.21(16) . . ?

Cl2B Zr1B C8B 79.88(11) . . ?

Cl1B Zr1B C8B 123.73(13) ..?

C3B $\operatorname{Zr1B}$ C8B $115.52(16)$. ?

C5B $\operatorname{Zr1B}$ C8B 143.94(16) . . ?

C7B Zr1B C10B 55.17(17) .. . ?

C1B Zr1B C10B 96.89(18) ...?

C2B $\operatorname{Zr} 1 \mathrm{~B}$ C10B $112.41(18)$. ?

$\mathrm{C} 6 \mathrm{~B} \quad \operatorname{zr} 1 \mathrm{~B}$ C10B $33.23(18)$. ?

Cl2B $\operatorname{Zr} 1 \mathrm{~B}$ ClOB $120.69(12) \cdots$ ?

Cl1B Zr1B C1OB 82.26(12)..?

C3B $\operatorname{Zr1B}$ C10B 145.77(17)..?

C5B $\operatorname{Zr} 1 \mathrm{~B}$ C10B $115.08(16)$. ?

C8B $\operatorname{Zr} 1 \mathrm{~B}$ C10B 52.66(16)..?

C7B ZI1B C4B 123.60(19)..?

$\mathrm{C} 1 \mathrm{~B} \quad \mathrm{Zr} 1 \mathrm{~B}$ C4B $54.68(17)$. ?

C2B Zr1B C4B 55.25(17) •.?

C6B Zr1B C4B 123.06(18) ..?

Cl2B Zr1B C4B 92.08(11)..?

Cl1B Zr1B C4B 89.17(11)..?

C3B Zr1B C4B 31.53(14). . ?

C5B $\operatorname{Zr} 1 \mathrm{~B}$ C4B 32.06(15) ..?

C8B $\operatorname{ar1B}$ C4B $147.02(16)$. ?

C10B Zr1B C4B 147.14(15). ?

C7B $\operatorname{Zr1B} C 9 \mathrm{~B} 53.2(2)$. ?

C1B $\operatorname{Zr1B}$ C9B 121.79(19) .. ?

C2B 2 I1B C9B 121.56(18) .. ?

C6B $\operatorname{Zr} 1 \mathrm{~B}$ C9B 53.42(18) . ?

Cl2B $\operatorname{Zr} 1 \mathrm{~B}$ C9B $90.52(12) \cdot . ?$

Cl1B ZriB C9B 92.78(13) .. ?

C3B $\operatorname{Zr1B}$ C9B $146.77(16)$. ?

C5B Zr1B C9B 145.44(16)..?

C8B $2 r 1 B$ C9B $31.37(16)$. ?

C10B Zr1B C9B $30.54(15)$. ? 
(C) 2004 American Chemical Society, J. Am. Chem. Soc., Baar ja040021j Supporting Info Page 17

C4B Zr1B C9B $176.36(18)$. ?

C11B SilB C12B 106.7(2)..?

C11B SilB C6B 115.2(2)..?

C12B Si1B C6B 117.3(2).: ?

C11B SilB C1B 107.1(2)..?

C12B SilB C1B 117.7(3)..?

C6B SilB C1B $92.5(2)$. ?

Cl1B SilB Zr1B 103.09(18) . . ?

C12B Si1B Zr1B 150.17(15) . . ?

C6B SilB Zr1B 48.78(16) ..?

C1B SilB Zr1B 49.13(18) . . ?

C14B Si2B C2B 106.9(2) •.?

C14B Si2B C13B 107.4(2) ..?

C2B Si2B C13B 118.2(2) . ?

C14B Si2B C7B $116.6(3)$. . ?

C2B Si2B C7B 92.8(2) . ?

C13B Si2B C7B 114.5(3) . . ?

C14B Si2B $\operatorname{Zr} 1 \mathrm{~B} 105.66(18)$. ?

C2B Si2B ZriB 48.82(18) : ?

C13B Si2B $\mathrm{Zr} 1 \mathrm{~B} 146.92(16)$. ?

C7B Si2B Zr1B 48.60(16) . . ?

C5B C1B C2B 107.4(4)..?

C5B C1B SilB 124.9(4) . ?

C2B C1B SilB $122.6(4)$. ?

C5B C1B $\operatorname{Zr} 1 \mathrm{~B} 78.4(4)$. ?

C2B C1B $2 r 1 B$ 72.5(3) . ?

Si1B C1B Zr1B 94.8(2) . ?

C3B C2B C1B 104.6(4)..?

$\mathrm{C} 3 \mathrm{~B} \mathrm{C} 2 \mathrm{~B} \mathrm{Si} 2 \mathrm{~B} 128.5(3)$. ?

C1B C2B Si2B 122.2(4) . . ?

C3B C2B Zr1B 77.2(3)..?

C1B C2B $\operatorname{Zr} 1 \mathrm{~B} 72.5(3)$. ?

Si2B C2B Zr1B 96.1(2) . ?

C4B C3B C2B 112.2(4)..?

C4B C3B $\operatorname{Zr} 1 \mathrm{~B} 78.0(3)$ : ?

C2B C3B Zr1B 69.4(3) . . ?

С 3 В С 4 В С5B $103.9(5)$. ?

С $3 \mathrm{~B}$ C4B C15B 131.1(4) . ?

C5B C4B C15B 124.9(4) . ?

С В $\mathrm{C} 4 \mathrm{~B}$ Zr1B 70.5(3) ..?

C5B C4B $2 r 1 B \quad 70.7(3)$. ?

C15B C4B Zr1B 125.1(4) . ?

C1B C5B C4B 111.9(4) . . ?

C1B C5B Zr1B 69.4(3) . . ?

C4B C5B Zr1B 77.3(3)..?

C7B C6B C10B 106.2(5)..?

C7B C6B SilB 122.9(4) . ?

C10B C6B SilB 127.0(3)..? 
(C) 2004 American Chemical Society, J. Am. Chem. Soc., Baar ja040021j Supporting Info Page 18

C7B C6B Zr1B $72.3(3) \cdot . ?$

$\mathrm{C} 10 \mathrm{~B}$ C6B $\operatorname{Zr} 1 \mathrm{~B} 80.8(3)$. ?

Si1B C6B Zr1B 95.4(2)..?

C8B C7B C6B 107.9(5) . ?

C8B C7B Si2B 127.3(3) •. ?

C6B C7B Si2B 120.8(4) . ?

C8B C7B $\mathrm{Zr} 1 \mathrm{~B} 80.6(3)$. ?

C6B C7B Zr1B $72.4(3) \cdot$ ?

Si2B C7B Zr1B 95.1(2) . ?

C7B C8B C9B 108.1(4)..?

С 7 B C8B C21B $126.7(5) . . ?$

С9B C8B C21B $125.0(5) . . ?$

C7B C8B Zr1B 67.0(3). ?

C9B C8B ZrIB $77.8(3) \cdot$. ?

$\mathrm{C} 21 \mathrm{~B}$ C8B Zr1B $124.7(3)$. ?

$\mathrm{C} 10 \mathrm{~B}$ C9B C8B $109.7(5) \cdot$ ?

$\mathrm{C} 10 \mathrm{~B}$ C9B $\mathrm{Zr} 1 \mathrm{~B} 72.2(3)$. ?

C 8 B C9B $2 r 1 B \quad 70.9(3)$. ?

C9B $\mathrm{C} 10 \mathrm{~B}$ C6B $108.0(5) . . ?$

C9B C10B C24B 125.1(5) •.?

C6B C 10B C24B 126.7(5) •.?

C9B C10B Zr1B 77.3(3) . ?

C6B C10B $\operatorname{Zr} 1 \mathrm{~B} 65.9(3)$. ?

C24B C10B Zr1B 126.4(3) . ?

C4B C15B C17B $111.6(5)$. ?

C4B C15B C16B $112.6(4) \cdot$ ?

CI7B C15B C16B $114.4(4)$. ?

C18B C17B C20B 108.0(4) . ?

$\mathrm{C} 18 \mathrm{~B} \quad \mathrm{Cl7B}$ C15B $110.8(4) \cdot . ?$

C20B C17B C15B $111.6(4) \cdot . ?$

C18B C17B C19B 108.4(4) . ?

C20B C17B C19B 109.3(4) •.?

C15B C17B C19B 108.7(5) . ?

C23B C21B C 8 B $112.7(4)$. ?

C23B C21B C22B 109.4(5) . ?

C 8 B C21B C22B $110.1(4)$. ?

C10B C24B C26B 113.4(4) . ?

C10B C24B C25B 109.6(4) . ?

C26B C24B C25B $109.5(5)$. ?

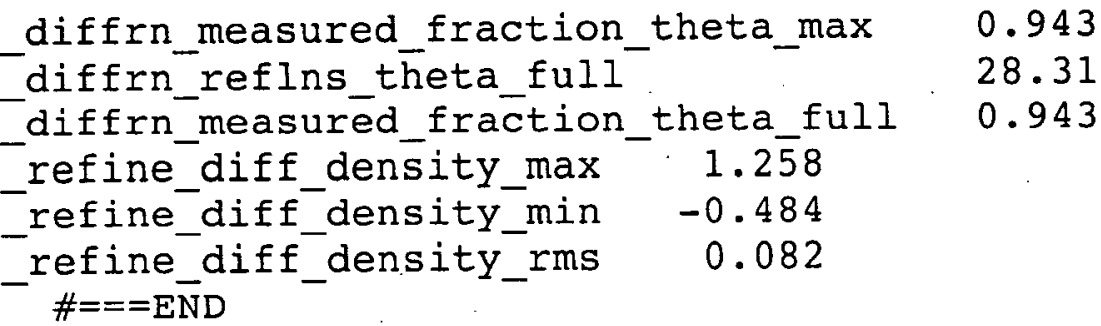


(C) 2004 American Chemical Society, J. Am. Chem. Soc., Baar ja040021j Supporting Info Page 19

CIF FILE FOR (S) -5

data_XXX \# cj15

_audit_creation_method

-database_code_CSD

SHELXL-97

chemical_name_systematic

146326

;

?

;

_chemical_name_common

chemical_melting_point

_chemical_formula_moiety

-chemical_formula_sum

_chemical_formula_weight

loop

atom_type_symbol

-atom_type_description

-atom_type_scat_dispersion_real

-atom_type_scat_dispersion_imag

atom_type_scat_source

${ }^{\prime} C^{\prime}{ }^{\prime}{ }^{\prime} C^{\prime}-0.00 \overline{3} 30.0016$

'International Tables Vol C Tables 4.2 .6 .8 and 6.1.1.4'

' $\mathrm{H}$ ' ' $\mathrm{H}$ ' $0.0000 \quad 0.0000$

'International Tables Vol C Tables 4.2 .6 .8 and 6.1.1.4'

'Si' 'Si' 0.08170 .0704

'International Tables Vol $\mathrm{C}$ Tables 4.2 .6 .8 and 6.1.1.4'

' $\mathrm{S}$ ' ' $\mathrm{S}$ ' $0.1246 \quad 0.1234$

'International Tables Vol C Tables 4.2 .6 .8 and 6.1.1.4'

' $\mathrm{zr}$ ' ' $\mathrm{zr}$ ' $-2.9673 \quad 0.5597$

'International Tables Vol C Tables 4.2 .6 .8 and 6.1.1.4'

_symmetry_cell_setting

_symmetry_space_group_name_H-M

_symmetry_Int_Tables_number

loop_

_symmetry_equiv_pos_as_xyz

$' \mathrm{x}, \mathrm{y}, \mathrm{z}$ '

' $-x,-y, z+1 / 2$ '

$'-y+1 / 2, x+1 / 2, z+3 / 4$ '

$' y+1 / 2,-x+1 / 2, z+1 / 4$ '

$'-x+1 / 2, y+1 / 2,-z+3 / 4$ '

$' x+1 / 2,-y+1 / 2,-z+1 / 4$ '

' $y, x,-z '$

$-y,-x,-z+1 / 2$ ' tetragonal

'P $43212^{\prime}$

96 
(C) 2004 American Chemical Society, J. Am. Chem. Soc., Baar ja040021j Supporting Info Page 20

$=$

_cell_length_a
_cell_length_b
_cell_length_c
_cell_angle_alpha
_cell_angle_beta
_cell_angle_gamma
-cell_volume

_cell_formula_units_z

_cell_measurement_temperature

_cell_measurement_reflns_used

_cell_measurement_theta_min

_cell_measurement_theta_max

exptl_crystal_description

-expt1_crystal_colour

-expt1_crystal_size_max

_expt1_crystal_size_mid

_expt1_crystal_size_min

-expt1_crystal_density_meas

_exptl_crystal_density_diffrn

-exptl_crystal_density method

exptl_crystal_F_000

expt1_absorpt_coefficient_mu

_expt 1_absorpt_correction_type

_exptl_absorpt_correction_T_min

_exptI_absorpt_correction_T_max

_expt__absorpt_process_detaíls

_expt1_special_details

?

;

diffrn_ambient_temperature

diffrn_radiation_wavelength

diffrn_radiation_type

diffrn_radiation_source

diffrn_radiation_monochromator

_diffrn_measurement_device_type

diffrn_measurement method

diffrn_detector_area_resol_mean

_diffrn_standards_number

_diffrn_standards_interval_time

_diffrn_standards_decay_o

-diffrn_reflns_number

_diffrn_reflns_av_R_equivalents

_diffrn_reflns_av_sigmaI/netI

_diffrn_reflns_limit_h_min

\author{
$12.6459(8)$ \\ $12.6459(8)$ \\ $47.844(4)$ \\ 90.00 \\ 90.00 \\ 90.00 \\ $7651.1(10)$ \\ 8
}

98

6207

2.3

27.0

'irregular block'

'pale yellow'

0.26

0.22

0.17

?

1.251

'not measured'

3040

0.483

none

?

?

?

98

0.71073

MoK\a

'normal-focus sealed tube'

graphite

'Bruker SMART $1000 \mathrm{ccd}$

' Iw scans at 3 fixed If values'

?

$?$

'first scans recollected at end of runs'

'within counting statistics'

75962

0.0820

0.0550

$-16$ 
(C) 2004 American Chemical Society, J. Am. Chem. Soc., Baar ja040021j Supporting Info Page 21

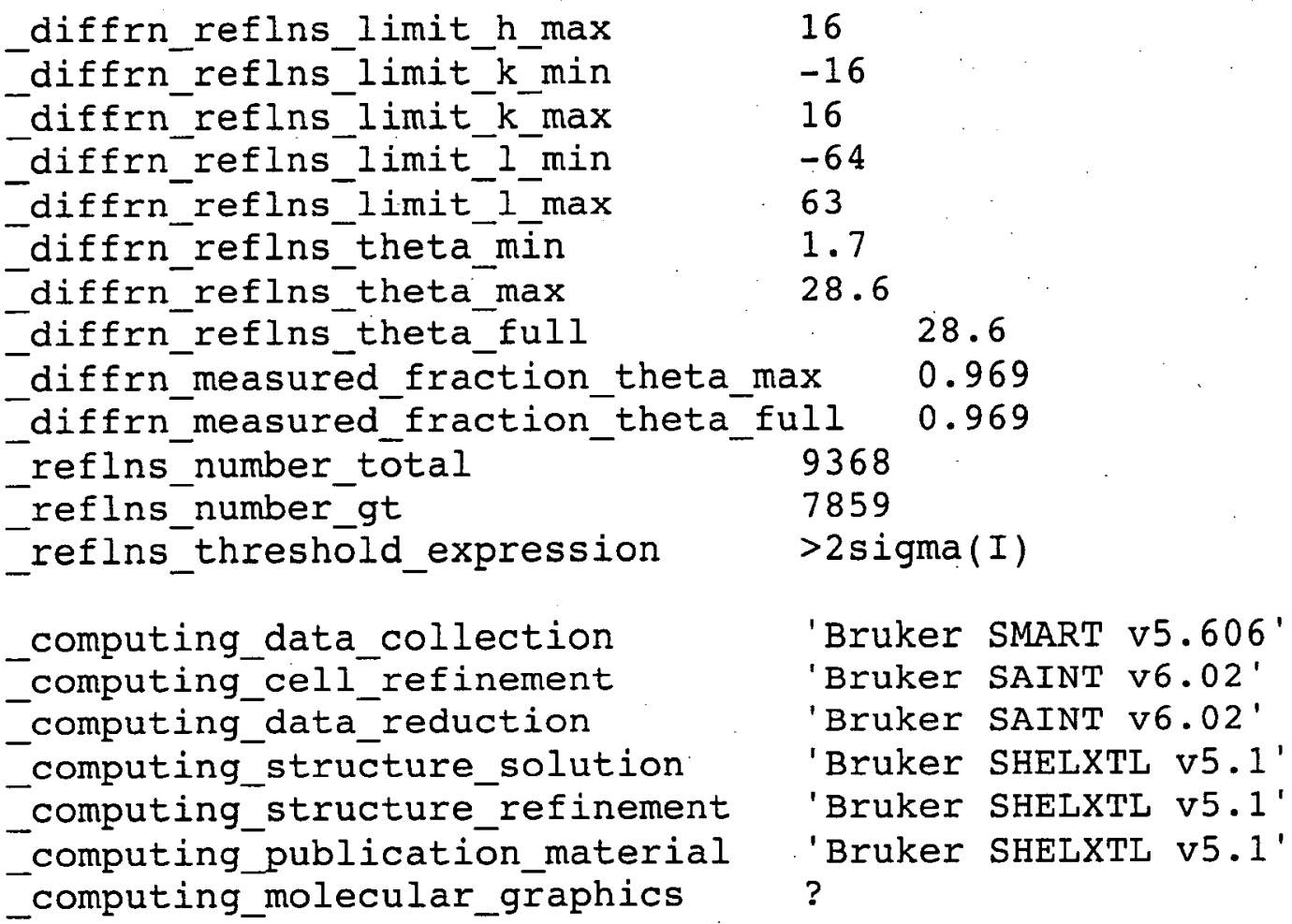

_refine_special_details

Refinement of $\mathrm{F}^{\wedge} 2^{\wedge}$ against all reflections. The weighted $\mathrm{R}$-factor $\mathrm{wR}$ and goodness of fit $S$ are based on $F^{\wedge} 2^{\wedge}$, conventional $R$-factors $R$ are based on $F$, with $F$ set to zero for negative $F^{\wedge} 2^{\wedge}$. The threshold expression of $F^{\wedge} 2^{\wedge}>2$ sigma $\left(F^{\wedge} 2^{\wedge}\right)$ is used only for calculating R-factors(gt) etc. and is not relevant to the choice of reflections for refinement. ;

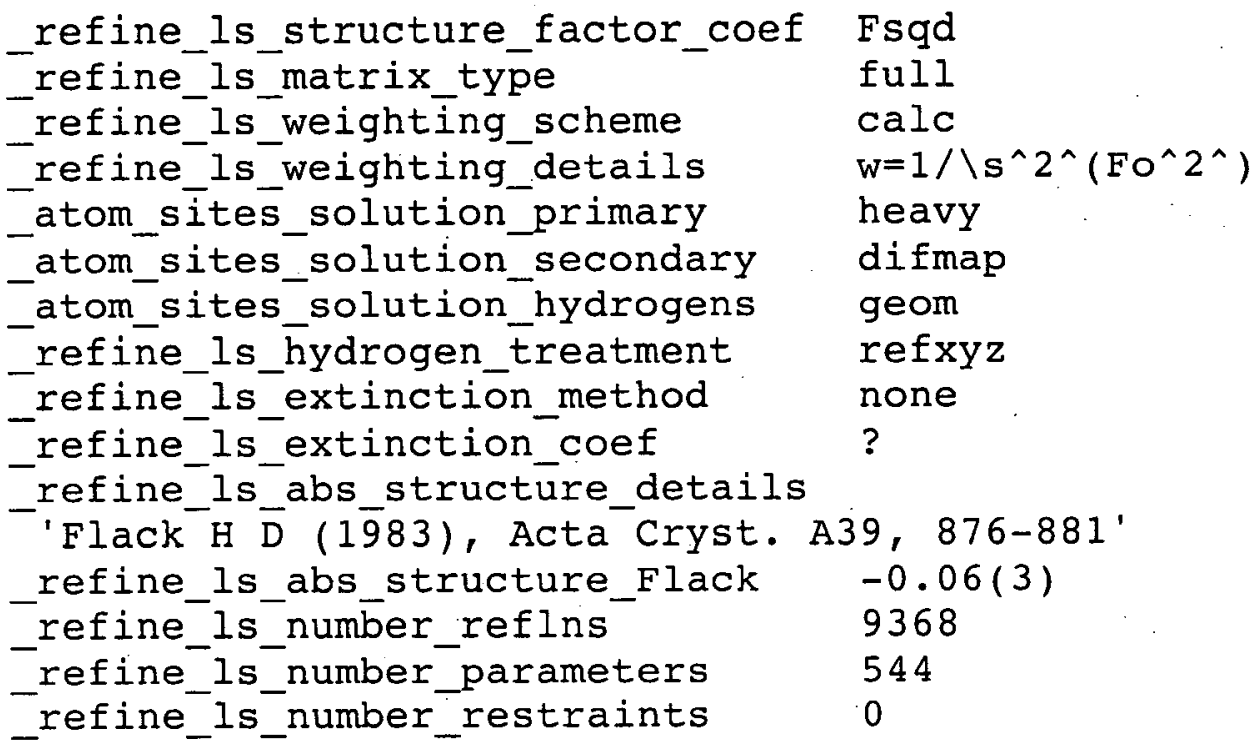


: (c) 2004 American Chemical Society, J. Am. Chem. Soc., Baar ja040021j Supporting Info Page 22

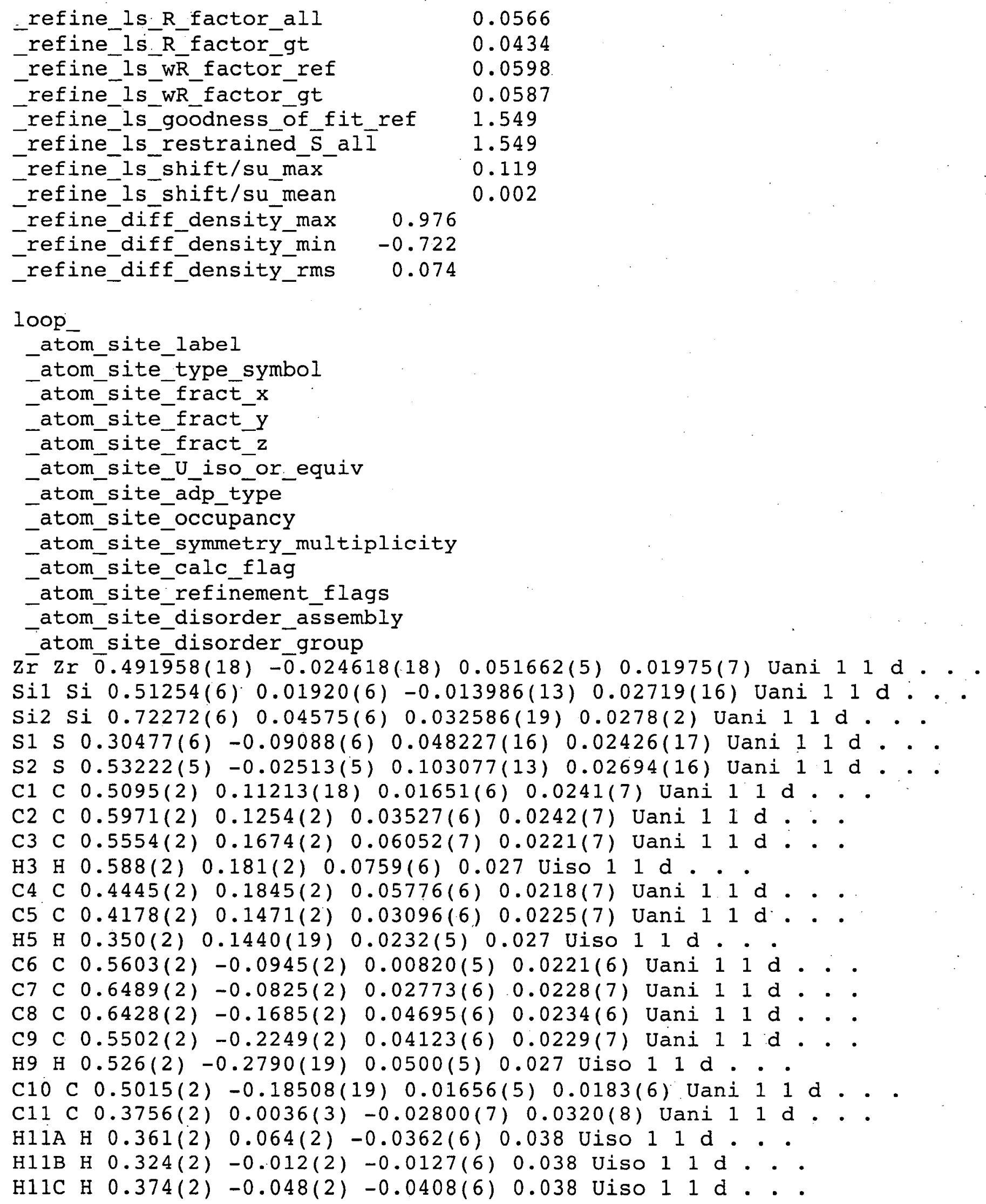


(C) 2004 American Chemical Society, J. Am. Chem. Soc., Baar ja040021j Supporting Info Page 23

C12 C $0.5973(3) 0.0601(3)-0.04343(7) \quad 0.0402(9)$ Uani $11 d$. H12A H $0.596(2) 0.023(3)-0.0575(6) 0.048$ Uiso $11 \mathrm{~d}$. . H12B H $0.666(2) 0.080(2)-0.0379(6) \quad 0.048$ Uiso I I d . . H12C H $0.578(2) 0.117(2)-0.0524(6) 0.048$ Uiso $11 \mathrm{~d}$. . C13 C $0.8181(3) \quad 0.0789(3) \quad 0.00434(9) \quad 0.0420(10)$ Uani $11 d$. . H13A H $0.834(3) 0.141(3) 0.0040(7) \quad 0.050$ Uiso $11 \mathrm{~d}$. . . H13B H $0.805(3) 0.063(3)-0.0138(7) 0.050$ Uiso $11 \mathrm{~d} .$. $\mathrm{H} 13 \mathrm{C} \mathrm{H} 0.884(3) \quad 0.049(2) \quad 0.0094(7) \quad 0.050$ Uiso $11 \mathrm{~d}$... C14 C $0.7926(3) \quad 0.0604(3) \quad 0.06637(8) \quad 0.0378(9)$ Uani $11 d$. . H1 $4 \mathrm{~A} \cdot \mathrm{H} \quad 0.850(2) \quad 0.020(2) \quad 0.0653(6) \quad 0.045$ Uiso $11 \mathrm{~d} .$. $\mathrm{H} 14 \mathrm{~B}$ H $0.753(2) \quad 0.042(2) \quad 0.0815(6) \quad 0.045$ Uiso $11 \mathrm{~d} .$. H14C H $0.807(2) \quad 0.139(2) \quad 0.0690(6) \quad 0.045$ Uiso 11 d . . C15 C $0.3727(2) \quad 0.2425(2) \quad 0.07774(6) \quad 0.0229(7) \quad \operatorname{Uani} 11 \mathrm{~d}$. . $\mathrm{H} 15 \mathrm{H} \quad 0.304(2) \quad 0.214(2) \quad 0.0742(5) \quad 0.027$ Uiso $11 \mathrm{~d} \ldots$. C16 C $0.4033(3) 0.2261(3) 0.10790(7) \quad 0.0329(8)$ Uani $11 \mathrm{~d}$. . H16A H $0.403(2) \quad 0.154(2) \quad 0.1131(6) \quad 0.039$ Uiso $11 \mathrm{~d}$. . . H16B H $0.353(2) \quad 0.259(2) \quad 0.1211(6) \quad 0.039$ Uiso $11 \mathrm{~d}$. . H16C H $0.468(2) \quad 0.250(2) \quad 0.1118(6) \quad 0.039$ Uiso 11 d . . . C17 C $0.3625(2) 0.3631(2) 0.06973(6) \quad 0.0255(7)$ Uani 11 d . . C18 C $0.3290(3) \quad 0.3744(3) \quad 0.03952(8) \quad 0.0375(9) \operatorname{Uani} 11 \mathrm{~d}$. . $\mathrm{H} 18 \mathrm{~A} \mathrm{H} 0.305(2) \quad 0.442(2) 0.0348(6) .0 .045$ Uiso $11 \mathrm{~d}$. . . H18B H $0.270(2) \quad 0.327(2) \quad 0.0341(7) .0 .045$ Uiso $11 \mathrm{~d}$. . H18C H $0.382(3) 0.359(2) 0.0274(7) \quad 0.045$ Uiso 11 d . . . C19 C $0.2763(3) \quad 0.4131(3) \quad 0.08799(8) \quad 0.0383(9)$ Uani $11 \mathrm{~d}$. . H19A H $0.267(2) 0.481(2) 0.0823(6) 0.046$ Uiso $11 \mathrm{~d}$. . . H19B H $0.299(2) 0.416(2) 0.1090(7) \quad 0.046$ Uiso 11 d . . . H19C H $0.204(3) \quad 0.376(2) \quad 0.0854(6) \quad 0.046$ Uiso 11 d.... C20 C $0.4658(3) \quad 0.4217(3) \quad 0.07424(9) \quad 0.0415(9) \operatorname{Uani} 11 \mathrm{~d}$. . H2OA H $0.517(2) 0.390(2) 0.0640(7) \quad 0.050$ Uiso $11 \mathrm{~d}$. H2OB H.0.489(3) $0.422(2) \quad 0.0931(7) \quad 0.050$ Uiso $11 \mathrm{~d}$. $\mathrm{H} 20 \mathrm{C}$ H $0.462(2) 0.496(2) \quad 0.0680(6) \quad 0.050$ Uiso 1 I d. C21 C $0.7289(2)-0.2038(2) \quad 0.06705(6) \quad 0.0298(7) \quad \operatorname{Uani} 11 \mathrm{~d}$... $\mathrm{H} 21 \mathrm{H} 0.741(2)-0.149(2) 0.0820(6) 0.036$ Uiso $11 \mathrm{~d}$. C22 C $0.8323(3)-0.2142(3) \quad 0.05050(8) 0.0385(9) \operatorname{Uani} 11 \mathrm{~d}$. $\mathrm{H} 22 \mathrm{~A} \mathrm{H} 0.890(2)-0.238(2) 0.0638(7) \quad 0.046$ Uiso $11 \mathrm{~d}$. . . H22B H $0.856(2)-0.149(3) 0.0423(7) \quad 0.046$ Uiso $11 \mathrm{~d}$ : . . H $22 \mathrm{C} \mathrm{H} 0.828(2)-0.256(2) \quad 0.0350(7) \quad 0.046$ Uiso $11 \mathrm{~d} .$. C23 C $0.7044(3)-0.3094(3) \quad 0.08104(7) \quad 0.0331(8)$ Uani 1 id . . $\mathrm{H} 23 \mathrm{~A} \mathrm{H} 0.636(2)-0.306(2) \quad 0.0937(6) 0.040$ Uiso $11 \mathrm{~d} .$. Н23B H $0.762(2)-0.322(2) 0.0916(6) 0.040$ Uiso: $11 \mathrm{~d}$. . . $\mathrm{H} 23 \mathrm{C} \mathrm{H} 0.698(2)-0.366(2) 0.0671(6) 0.040$ Uiso $11 \mathrm{~d}$. . . C24 C $0.4134(2)-0.2386(2) \quad 0.00059(7) \quad 0.0280(8)$ Uani $11 d$. $\mathrm{H} 24 \mathrm{H} 0.362(2)-0.194(2)-0.0022(6) 0.034$ Uiso $11 \mathrm{~d}$. . . C25 C $0.4548(3)-0.2741(3)-0.02775(7) \quad 0.0392(9)$ Uani $11 d$.. H25A H $0.482(2)-0.217(2)-0.0397(6) 0.047$ Uiso $1.1 \mathrm{~d}$. . $\mathrm{H} 25 \mathrm{~B} \mathrm{H} 0.395(2)-0.305(2)-0.0384(7) 0.047$ Uiso $11 \mathrm{~d} .$. $\mathrm{H} 25 \mathrm{C} \mathrm{H} 0.514(2)-0.321(2)-0.0249(6) \quad 0.047$ Uiso 11 d... C26 C $0.3680(3)-0.3344(3) \quad 0.01605(8) \quad 0.0345(8)$ Uani $11 d$. . 
(C) 2004 American Chemical Society, J. Am. Chem. Soc., Baar ja040021j Supporting Info Page 24

H 26A H $0.426(2)-0.389(2) \quad 0.0178(6) 0.041$ Uiso $11 \mathrm{~d} \cdot$. H26B H $0.309(2)-0.358(2) \quad 0.0060(6) \quad 0.041$ Uiso $11 \mathrm{~d} .$. $\mathrm{H} 26 \mathrm{C} \mathrm{H} 0.346(2)-0.320(2) 0.0340(7) 0.041$ Uiso $11 \mathrm{~d} . .$. C27 C $0.2198(2) 0.0047(2) 0.06358(6) 0.0256(6)$ Uani 11 d . . C28 C $0.1464(2) \quad 0.0559(2) 0.04644(7) 0.0304(7)$ Uani $11 \mathrm{~d} .$. H28 H $0.143(2) 0.038(2) 0.0263(6) 0.036$ Uiso $11 \mathrm{~d} .$. C29 C $0.0762(3) 0.1275(3) 0.05797(9) 0.0428(10)$ Uani 11 d . . H 29 H $0.032(2) 0.164(2) 0.0462(6) 0.051$ Uiso 1 l d . .

C30 C $0.0788(3) 0.1492(3) 0.08586(9) 0.0460(11)$ Uani $11 \mathrm{~d} .$. H30 H $0.035(3) \quad 0.193(2) 0.0940(7) \quad 0.055$ Uiso $11 \mathrm{~d} .$. C31 C $0.1499(3) 0.0986(3) 0.10316(8) 0.0419(9)$ Uani $11 d .$. $\mathrm{H} 31 \mathrm{H} 0.156(2) 0.112(3) 0.1219(7) 0.050$ Uiso $11 \mathrm{~d} .$. C32 C $0.2208(2) 0.0255(3) \quad 0.09178(7) 0.0327(8)$ Uani 11 d . . H32 H $0.267(2)-0.005(2) 0.1028(6) \quad 0.039$ Uiso $11 \mathrm{~d} . .$. C33 C $0.5224(2)-0.1538(2) 0.11757(6) 0.0249(7)$ Uani 11 d . . C34 C $0.5962(3)-0.1836(2) 0.13771(6) 0.0294(7)$ Uani $11 d$. . H34 H $0.653(2)-0.141(2) 0.1423(6) 0.035$ Uiso $11 \mathrm{~d} . .$. C35 C $0.5903(3)-0.2824(3) 0.15008(7) 0.0363(9)$ Uani $11 \mathrm{~d}$. . H 35 H $0.646(2)-0.297(2) \quad 0.1630(6) \quad 0.044$ Uiso $11 \mathrm{~d} . .$. C36 C $0.5109(3)-0.3518(2) 0.14320(7) 0.0372(8)$ Uani 11 d . . H36 H $0.507(2)-0.417(2) 0.1519(6) \quad 0.045$ Uiso $11 \mathrm{~d} .$. C37 C $0.4362(3)-0.3224(3) 0.12363(7) 0.0341(8)$ Uani $11 \mathrm{~d} .$. H37 H $0.385(2)-0.357(2) 0.1185(6) 0.041$ Uiso $11 \mathrm{~d} . .$. C38 C $0.4406(2)-0.2230(2) 0.11085(7) 0.0288(8)$ Uani $11 \mathrm{~d}$. . $\mathrm{H} 38 \mathrm{H} 0.395(2)-0.201(2) 0.0983(6) 0.035$ Uiso $11 \mathrm{~d} .$.

\section{loop}

_atom_site_aniso_label

-atom_site_aniso_U_11

-atom_site_aniso_U_22

-atom_site_aniso_U_33

-atom_site_aniso_u_23

atom_site_aniso_U_13

atom_site_aniso_U_12

$\mathrm{z} \overline{\mathrm{r}} 0.0 \overline{1} 816(\overline{1} 4) \quad 0 . \overline{0} 1 \overline{9} 10(13) \quad 0.02200(12) \quad 0.00083(12) \quad 0.00175(10) \quad 0.00103(11)$

Sil $0.0326(4) \quad 0.0242(4) \quad 0.0247(4) 0.0022(3) \quad 0.0054(3) 0.0049(4)$

Si2 $0.0218(4) \quad 0.0254(5) \quad 0.0363(5)-0.0005(4) \quad 0.0055(4) 0.0003(4)$

S1 $0.0205(4) 0.0243(4) 0.0280(4)-0.0021(4) 0.0017(3) 0.0000(3)$

S2 $0.0332(4) 0.0231(3) 0.0245(3) 0.0006(3)-0.0017(3) 0.0024(3)$

C1 $0.0301(18) 0.0144(13) 0.0278(16) 0.0074(11) 0.0041(15) 0.0010(13)$

$\begin{array}{llllllll}C 2 & 0.0233(16) & 0.0189(16) & 0.0304(17) & 0.0034(14) & 0.0046(14) & -0.0007(11)\end{array}$

C3 $0.0234(17) \quad 0.0173(15) \quad 0.0258(18) \quad 0.0002(13) 0.0010(14)-0.0038(12)$

C4 $0.0251(16) 0.0163(15) 0.0239(17) 0.0088(12) 0.0040(13) 0.0003(12)$

C5 $0.0259(16) \quad 0.0175(15) \quad 0.0242(17) \quad 0.0044(13)-0.0018(14) \quad 0.0042(13)$

C6 $0.0275(16) \quad 0.0223(15) \quad 0.0163(15)-0.0004(12) \quad 0.0045(12) \quad 0.0048(11)$

C7 $0.0140(14) 0.0251(16) \quad 0.0292(18)-0.0029(13) \quad 0.0090(13) 0.0015(12)$

C8 $0.0238(16) \quad 0.0213(15) \quad 0.0251(16)-0.0047(13) \quad 0.0010(13) \quad 0.0073(11)$

C9 $0.0283(17) 0.0171(15) 0.0233(17) 0.0040(12) 0.0066(13) 0.0022(12)$ 
(C) 2004 American Chemical Society, J. Am. Chem. Soc., Baar ja040021j Supporting Info Page 25

C10 $0.0204(15) \quad 0.0220(13) 0.0126(14)-0.0047(11) 0.0061(13) 0.0013(12)$

C11 $0.0435(18) \quad 0.0295(19) \quad 0.0229(18)-0.0005(15)-0.0108(15) \quad 0.0087(17)$

C12 $0.059(2) \quad 0.036(2) \quad 0.0252(19) \quad 0.0076(15) 0.0119(18) 0.0083(18)$

C13 $0.036(2) \quad 0.040(2) 0.050(2) \quad 0.001(2) 0.017(2)-0.0063(18)$

C14 $0.0234(19) \quad 0.035(2) \quad 0.056(3)-0.0034(19)-0.0017(17) \quad 0.0006(16)$

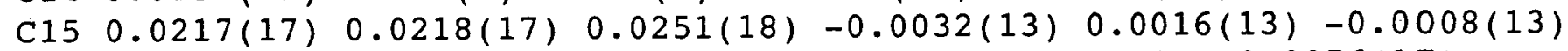

$\begin{array}{llllllll}\mathrm{C} 16 & 0.039(2) & 0.036(2) & 0.0242(19) & -0.0045(16) & 0.0039(17) & 0.0076(17)\end{array}$

C17 $0.0290(17) \quad 0.0214(16) \quad 0.0261(19) \quad-0.0029(14) \quad 0.0052(14) \quad 0.0023(14)$

C18 $0.049(2) \quad 0.0245(19) 0.039(2) 0.0024(16) 0.0006(18) 0.0114(17)$

$\begin{array}{lllllll}\text { C19 } & 0.049(2) & 0.0277(19) & 0.038(2) & -0.0041(17) & 0.0064(19) & 0.0074(17)\end{array}$

C20 $0.038(2) 0.0253(19) 0.061(3)-0.0016(18) 0.001(2)-0.0031(17)$

C21 $0.0289(18) \quad 0.0301(19) \quad 0.0304(18)-0.0029(15)-0.0039(15) \quad 0.0096(14)$

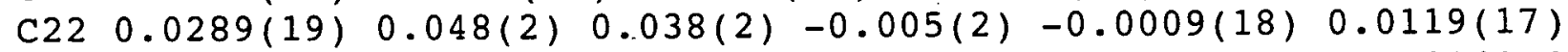

C23 $0.036(2) \cdot 0.0312(19) 0.032(2)-0.0031(16)-0.0031(16) 0.0141(16)$

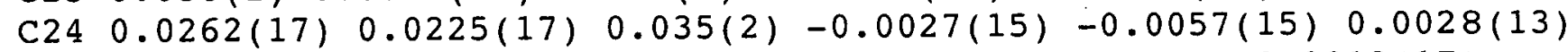

C25 $0.045(2) \quad 0.039(2) \quad 0.034(2)-0.0059(17) 0.0009(18)-0.0012(17)$

$\begin{array}{lllllll}\mathrm{C} 26 & 0.035(2) & 0.035(2) & 0.033(2) & -0.0001(17) & -0.0006(18) & -0.0048(16)\end{array}$

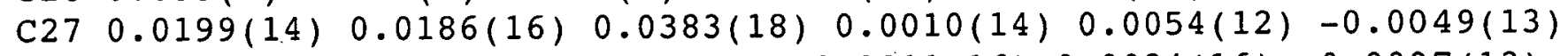

C28 $0.0206(15) \quad 0.0289(17) \quad 0.042(2)-0.0011(16) 0.0034(16)-0.0007(13)$

C29 $0.031(2) \quad 0.033(2) \quad 0.065(3) 0.0069(19) 0.0081(19) 0.0012(16)$

C30 $0.034(2) \quad 0.031(2) \quad 0.074(3)-0.008(2) 0.026(2)-0.0037(17)$

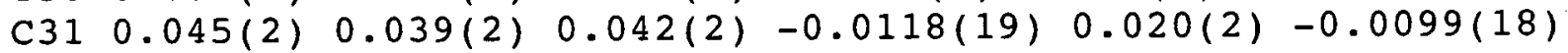

C32 $0.0312(18) 0.0324(18) 0.035(2) 0.0036(16) 0.0105(14)-0.0051(16)$

$\begin{array}{llllllll}\mathrm{C} 33 & 0.0335(17) & 0.0219(14) & 0.0192(15) & -0.0043(12) & 0.0016(14) & 0.0099(14)\end{array}$

$\begin{array}{llllllll}\text { C34 } & 0.0352(19) & 0.0326(19) & 0.0203(17) & -0.0036(14) & 0.0008(15) & 0.0090(14)\end{array}$

C35 $0.048(2) 0.039(2) 0.0221(19) 0.0019(16) 0.0003(16) 0.0156(18)$

C36 $0.049(2) 0.0270(16) 0.0351(19) 0.0076(15) 0.0069(18) 0.0072(18)$

C37 $0.032(2) 0.028(2) 0.042(2) \quad 0.0020(16) 0.0081(17)-0.0025(15)$

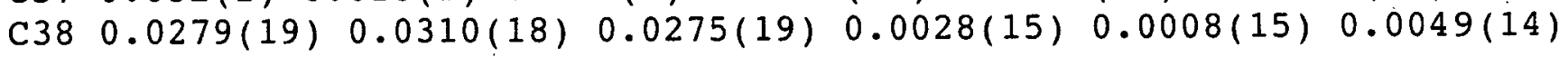

_geom_special_details

;

All esds (except the esd in the dihedral angle between two l.s. planes) are estimated using the full covariance matrix. The cell esds are taken into account individually in the estimation of esds in distances, angles and torsion angles; correlations between esds in cell parameters are only used when they are defined by crystal symmetry. An approximate

(isotropic)

treatment of cell esds. is used for estimating esds involving 1.s. planes. ;

loop

_geom_bond_atom_site_label_1

_geom_bond_atom_site_label_2

_geom_bond_distance

_geom_bond_site_symmetry_2

-geom_bond_publ_flag

$\mathrm{z} \overline{\mathrm{r}} \mathrm{CpA} 2.24 \overline{8}$. ? 


\begin{tabular}{|c|c|c|}
\hline & СpB & \\
\hline$r$ & $\mathrm{P} \ln \mathrm{A} 2.224(1)$ & \\
\hline & $P \ln B 2.226(1)$ & - \\
\hline & C7 $2.406(3)$. & ? \\
\hline & $2.419(2)$ & $?$ \\
\hline & $2.423(2)$ & $?$ \\
\hline & C2 $2.446(3)$ & ? \\
\hline & C5 $2.565(3)$ & ? \\
\hline & C3 $2.592(3)$. & $?$ \\
\hline & C10 2.637(2) & . ? \\
\hline & C8 $2.645(2)$. & - ? \\
\hline & C9 $2.684(3)$ & ? \\
\hline & C4 $2.728(3)$ & . ? \\
\hline & s2 $2.5120(7)$ & . ? \\
\hline & S1 $2.516 .5(8)$ & . ? \\
\hline & $\mathrm{C} 12 \cdot 1.844(3)$ & \\
\hline & C11 1.867 & \\
\hline i1 & C1 $1.874(3)$ & . ? \\
\hline 11 & C6 $1.887(3)$ & \\
\hline & $\mathrm{C} 14 \quad 1.852(4)$ & \\
\hline i2 & C13 1.859(4) & \\
\hline 12 & C7 $1.885(3)$ & \\
\hline i2 & 2 C2 $1.886(3)$ & - ? \\
\hline & C27 $1.777(3)$ & \\
\hline & C33 1.773(3) & . ? \\
\hline & C5 $1.420(4)$. & . ? \\
\hline & C2 $1.436(4)$ & $?$ \\
\hline & C3 $1.420(4)$ & ? \\
\hline & C4 $1.426(4)$ & - ? \\
\hline & $\mathrm{H} 30.86(3)$. & $?$ \\
\hline & C5 $1.407(4)$ & - ? \\
\hline & C15 1.509(4) & \\
\hline & H5 $0.93(3)$. & $?$ \\
\hline & $C 101.423(4)$ & ? ? \\
\hline & C7 $1.467(4)$ & \\
\hline$: 7$ & C8 $1.426(4)$ & . ? \\
\hline c8 & C9 $1.397(4)$ & . ? \\
\hline 8 & C21 $1.520(4)$ & \\
\hline & C10 $1.423(4)$ & \\
\hline & H9 $0.86(3)$ & $?$ \\
\hline & OC24 1.511(4 & \\
\hline & $1 \mathrm{H} 11 \mathrm{~A} \quad 0.87$ (3 & \\
\hline & $1 \mathrm{H} 11 \mathrm{~B} \quad 1.00(3$ & \\
\hline & $1 \mathrm{H} 11 \mathrm{C} 0.9013$ & ) \\
\hline & $\mathrm{H} 12 \mathrm{~A} \quad 0.82(3$ & \\
\hline & $\mathrm{H} 12 \mathrm{~B} \quad 0.95$ (3 & \\
\hline & $\mathrm{H} 12 \mathrm{C}$ & \\
\hline & H13A $0.80(3$ & \\
\hline
\end{tabular}




\begin{tabular}{|c|c|c|c|}
\hline & DIIJ & & \\
\hline & H13C & $0.95(3)$ & \\
\hline & $\mathrm{H} 14 \mathrm{~A}$ & & \\
\hline & $\mathrm{H} 14 \mathrm{~B}$ & & \\
\hline & H $14 \mathrm{C}$ & $1.02(3)$ & \\
\hline & $\mathrm{C} 16$ & & \\
\hline & $\mathrm{C} 17$ & & \\
\hline & H 15 & 0.96 & \\
\hline 16 & $\mathrm{H} 16 \mathrm{~A}$ & & \\
\hline & $\mathrm{H} 16 \mathrm{~B}$ & & \\
\hline & $\mathrm{H} 16 \mathrm{C}$ & & \\
\hline & C18 & & \\
\hline & & & \\
\hline & C19 & 1.5 & \\
\hline 8 & H $18 \mathrm{~A}$ & & \\
\hline & H $18 B$ & & \\
\hline & H18C & 0.5 & \\
\hline & H19A & & \\
\hline & $\mathrm{H} 1$ & & \\
\hline & $\mathrm{H} 1 \mathrm{~S}$ & 1.0 & \\
\hline & $\mathrm{H} 20 \mathrm{~A}$ & & \\
\hline & $\mathrm{H} 2 \mathrm{OB}$ & & \\
\hline & $\mathrm{H} 2 \mathrm{OC}$ & 0.5 & \\
\hline 21 & $\mathrm{C} 23$ & 1.5 & \\
\hline & $\mathrm{C} 22$ & 1.5 & \\
\hline & $\mathrm{H} 21$ & 1.0 & \\
\hline 22 & $\mathrm{H} 22 \mathrm{~A}$ & & \\
\hline 22 & $\mathrm{H} 22 \mathrm{~B}$ & 30. & \\
\hline C22 & $\mathrm{H} 22 \mathrm{C}$ & 0.9 & \\
\hline 23 & H23A & & \\
\hline$C 23$ & $\mathrm{H} 23 \mathrm{~B}$ & 30.9 & \\
\hline $\mathrm{C} 23$ & $\mathrm{H} 23 \mathrm{C}$ & 0.9 & \\
\hline$C 24$ & C25 & 1.52 & \\
\hline $\mathrm{C} 24$ & $\mathrm{C} 26$ & 1.5 & \\
\hline $\mathrm{C} 24$ & $\mathrm{H} 24$ & 0.87 & \\
\hline C25 & $\mathrm{H} 25 \mathrm{~A}$ & & \\
\hline 25 & $\mathrm{H} 25 \mathrm{~B}$ & 30.5 & \\
\hline C25 & $\mathrm{H} 25 \mathrm{C}$ & $=0.9$ & \\
\hline & $\mathrm{H} 26 \mathrm{~A}$ & 1.0 & \\
\hline & $\mathrm{H} 26 \mathrm{~B}$ & 30.5 & \\
\hline $\mathrm{C} 26$ & $\mathrm{H} 26 \mathrm{C}$ & 0.9 & \\
\hline & C 32 & & \\
\hline & $\mathrm{C} 28$ & 1.3 & \\
\hline $\mathrm{C} 28$ & C29 & 1.38 & \\
\hline & H 28 & & \\
\hline & C30 & & \\
\hline & H 29 & 0.92 & \\
\hline & & & \\
\hline
\end{tabular}




\begin{tabular}{|c|c|c|c|}
\hline 30 & $\mathrm{H} 30$ & & \\
\hline 31 & C32 & $1.399(4)$ & \\
\hline 031 & H 31 & $0.92(3)$ & \\
\hline C32 & H32 & $0.88(3)$ & \\
\hline 33 & C38 & $1.392(4)$ & \\
\hline C33 & C34 & $1.394(4)$ & \\
\hline C34 & C35 & 1.3 & \\
\hline C34 & H 34 & $0.92(3)$ & \\
\hline C35 & C36 & $1.373(5)$ & \\
\hline C35 & H35 & $0.96(3)$ & \\
\hline C36 & C37 & $1.381(5)$ & \\
\hline C36 & H36 & $0.92(3)$ & \\
\hline C37 & C38 & $1.399(4)$ & \\
\hline C37 & H3 7 & 0.8 & \\
\hline C38 & H38 & $0.88(3)$ & \\
\hline
\end{tabular}

100p_ _geom_angle_atom_site_label_1 -geom_angle_atom_site_label_2 _geom_angle_atom_site_label_3 -geom_angle _geom_angle_site_symmetry_1 _geom_angle_site_symmetry_3 _geom_angle_publ_flag $\overline{\mathrm{CPA}} \mathrm{Zr} \mathrm{CPB} 121.9 \div$ ? CpA Zr S1 112.0 . . ? CPA Zr S2 103.1..? CpB Zr S1 101.5 ..? CpB Zr S2 112.7 . . ? S2 $\operatorname{Zr~S1} 104.70(3)$. ? PlnA . PlnB 75.76(9) . ?

C12 Sil C11 107.12(17) . ?

C12 Si1 C1 115.52(15) . ?

C11 Sil C1 $109.06(14)$. . ?

C12 Si1 C6 117.22(15) . . ?

C11 Si1 C6 114.75(14) . . ?

C1 Si1 C6 92.67(11) . ?

C14 Si2 C13 107.60(18) . ?

C14 Si2 C7 115.47(15) . . ?

C13 Si2 C7 115.19(15) . ?

C14 Si2 C2 106.81(15) . . ?

C13 Si2 C2 118.39(16) . . ?

C7 Si2 C2 92.92(11)..?

C27 S1 Zr 108.40(9) ..?

C33 S2 $\operatorname{zr} 111.78(9) \cdot$. ?

C5 C1 C2 106.8(2) . ?

C5 C1 Sil 126.3(2) . . ?

C2 C1 Sil 123.0(2) . . ? 
(C) 2004 American Chemical Society, J. Am. Chem. Soc., Baar ja040021j Supporting Info Page 29

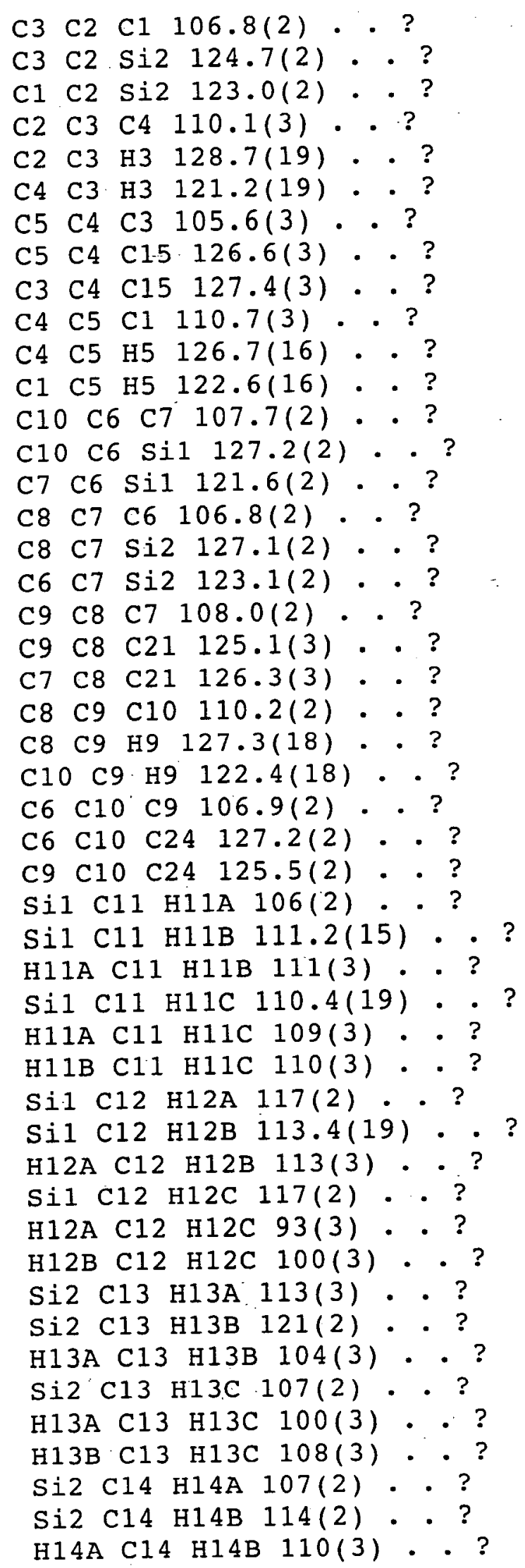


Si2 C14 H14C 107.2(18) . ?

H14A C14 H14C 114(3) •.?

$\mathrm{H} 14 \mathrm{~B} \mathrm{C} 14 \mathrm{H} 14 \mathrm{C} 105(3) \cdot . ?$

C16 C15 C4 $112.6(2) \cdot \cdot$ ?

C16 $\mathrm{C} 15 \mathrm{C} 17 \quad 112.8(3)$. ?

C4. C15 C17 $111.4(2)$. . ?

C16 C15 H15 $110.4(16)$. ?

C4 C15 H15 104.3(15) . ?

C17 C15 H15 104.6(15) . ?

C15 C16 H16A 112.7(18) . ?

C15 C16 H16B 112.8(18) . ?

H16A C16 H16B 103(2) . ?

C15 C16 H16C 113(2) . . ?

H16A C16 H16C $106(3) \ldots$ ?

H16B C16 H16C $109(3) .$. ?

$\begin{array}{llllll}\mathrm{C} 18 & \mathrm{C} 17 & \mathrm{C} 20 & 109.3(3) & . & \text {. ? }\end{array}$

$\begin{array}{lllll}\mathrm{C} 18 & \mathrm{C} 17 & \mathrm{C} 19 & 107.9(3) & .\end{array}$

$\begin{array}{lllll}\mathrm{C} 20 & \mathrm{C} 17 & \mathrm{C} 19 & 109.2(3) & .\end{array}$

$\begin{array}{lllll}\mathrm{C} 18 & \mathrm{C} 17 & \mathrm{C} 15 & 110.3(3) & .\end{array}$

C20 C17 C15 $111.5(3)$. . ?

$\begin{array}{llllll}\mathrm{C} 19 & \mathrm{C} 17 & \mathrm{C} 15 & 108.6(3) & \text {. ? ? }\end{array}$

C17 C18 H18A 114.2(19) . ?

C17 C18 H18B $113.9(19) \cdot$ ?

H18A C18 H18B $104(2) \cdot . ?$

$\mathrm{C} 17 \mathrm{C} 18 \mathrm{H} 18 \mathrm{C} \quad 113(2) \cdot . ?$

H18A C18 H18C $106(3)$.. ?

H18B C18 H18C 105(3) . ?

C17 C19 H19A 108.2(19) •. ?

C17 C19 H19B $111.5(17)$. ?

H19A C19 Н19B 107(2) •.?

C17 C19 H19C $112.0(17) \cdot$ ?

H19A C19 H19C 106(2)..?

H19B C19 H19C $112(2) \cdot$. ?

C17 C20 H20A $109(2) \cdot . ?$

$\mathrm{C} 17 \mathrm{C} 20$ H $20 \mathrm{~B} 113(2) \cdot \therefore$ ?

$\mathrm{H} 20 \mathrm{~A} \mathrm{C} 20 \mathrm{H} 2 \mathrm{OB} 107(3)$. ?

C 17 C20 H20C $112.2(18)$. ?

$\mathrm{H} 20 \mathrm{~A}$ C20 H2OC $107(3)$. ?

H2OB C2O H2OC $108(2): . ?$

$\begin{array}{llllll}\mathrm{C} 8 & \mathrm{C} 21 & \mathrm{C} 23 & 112.9(3) & .\end{array}$

$\begin{array}{llllll}\mathrm{C} 8 & \mathrm{C} 21 & \mathrm{C} 22 & 108.0(3) \cdot . ?\end{array}$

C23 C21 C22 $108.9(3) \cdot$ ?

C 8 C21 H21 $110.6(16)$. ?

$\mathrm{C} 23 \mathrm{C} 21 \mathrm{H} 21 \mathrm{108.8(16)} \mathrm{.} \mathrm{?}$

C22 C21 H21 $107.4(17) \cdot$ ?

$\mathrm{C} 21 \mathrm{C} 22 \mathrm{H} 22 \mathrm{~A} 108.1(18) \cdot \cdot$ ?

$\mathrm{C} 21 \mathrm{C} 22 \mathrm{H} 22 \mathrm{~B} 113.6(19) \cdot$ ?

$\mathrm{H} 22 \mathrm{~A}$ C 22 H22B $107(3)$. ? 


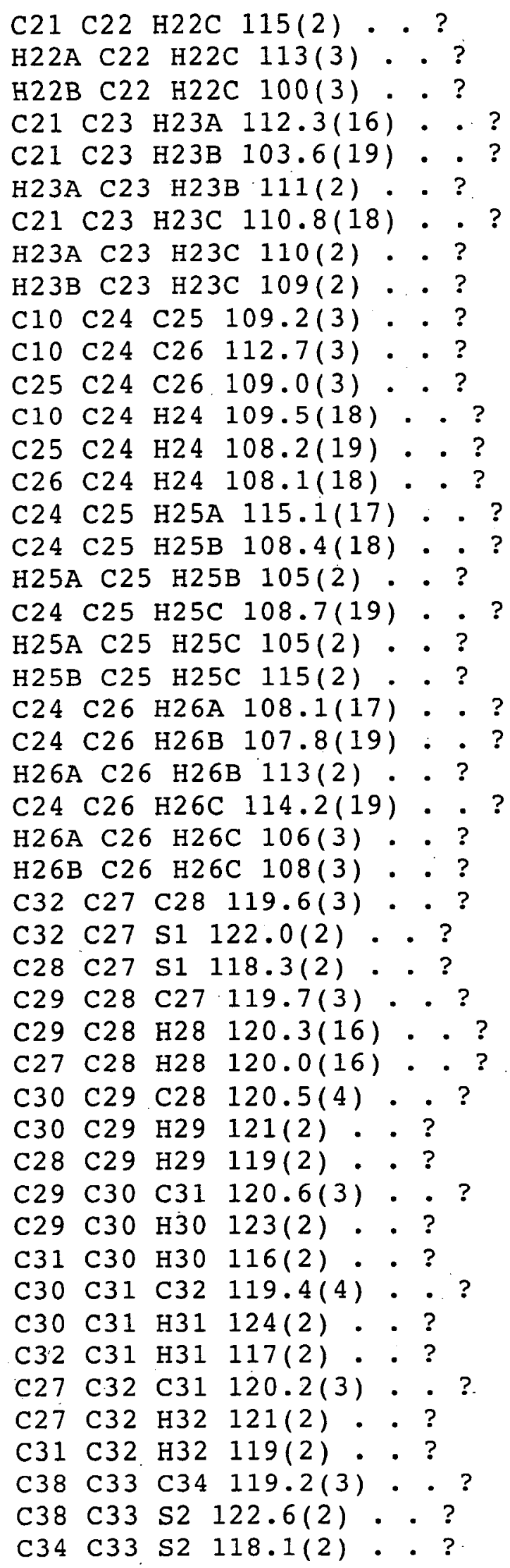


(C) 2004 American Chemical Society, J. Am. Chem. Soc., Baar ja040021j Supporting Info Page 32

\begin{tabular}{|c|c|c|c|c|}
\hline 35 & C34 & C33 & $120.2(3)$ & \\
\hline C35 & C34 & H 34 & $117.5(18)$ & \\
\hline C33 & C34 & H34 & $122.1(18)$ & \\
\hline C36 & C35 & C34 & $120.9(3)$ & \\
\hline C36 & C35 & H35 & $124.6(19)$ & \\
\hline C34 & C35 & H35 & $114.4(19)$ & \\
\hline C35 & C36 & C37 & $119.4(3)$ & \\
\hline C35 & C36 & H36 & $120(2)$. & \\
\hline C37 & C36 & H 36 & $121(2)$ & . \\
\hline C36 & C37 & C38 & $120.7(3)$ & \\
\hline C36 & C37 & H 37 & $127(2)$. & \\
\hline C38 & C37 & H37 & $113(2)$. & \\
\hline C33 & C38 & C37 & $119.6(3)$ & \\
\hline C33 & C38 & H38 & $117(2)$. & \\
\hline C3 & C38 & H38 & $124(2)$ & \\
\hline
\end{tabular}

loop _geom_torsion_atom_site_label_1 _geom_torsion_atom_site_label_2 _geom_torsion_atom_site_label_3 -geom_torsion_atom_site_label_4 -geom_torsion -geom_torsion_site_symmetry_1 _geom_torsion_site_symmetry_2 -geom_torsion_site_symmetry_3 -geom_torsion_site_symmetry_4 _geom_torsion_publ_flag $s \overline{2} \operatorname{Zr} \bar{s} 1$ C27 $6 \overline{6} .87(\overline{1} 1)$... ? $\mathrm{S} 1 \mathrm{zr}$ S2 C33 60.65(11) ... ? C12 Si1 C1 C5 133.8(3) . . . ? C11 Sil C1 C5 13.2(3)....? C6 Si1 C1 C5 $-104.2(2) \cdot .$. ? C12 Si1 C1 C2 $-71.7(3) . .$. ? C11 Sil C1 C2 167.7(2)....? C6 Sil C1 C2 50.3(2) . . . ?

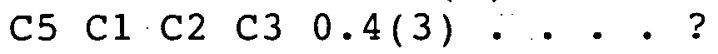
Sil C1 C2 C3 $-158.4(2) . .$. ? C5 C1 C2 Si2 155.4(2) ....? Si1 C1 C2 Si2 $-3.3(3) \cdot . . \cdot$ ? C14 Si2 C2 C3 $-12.6(3) \cdot . . . ?$ C13 Si2 C2 C3 -134.1(3)... ? C7 Si2 C2 C3 105.1(2) ....? C14 Si2 C2 C1 $-163.2(2) . . . . ?$ $\mathrm{C} 13 \mathrm{Si2}$ C2 C1 75.3(3) . . : ? C7 Si2 C2 C1 $-45.5(2) . . . \therefore$ ? C1 C2 C3 C4 $-2.2(3)$. . . ? Si2 C2 C3 C4 $-156.7(2) \cdot . . . ?$ C2 C3 C4 C5 3.1(3) . . . ? 


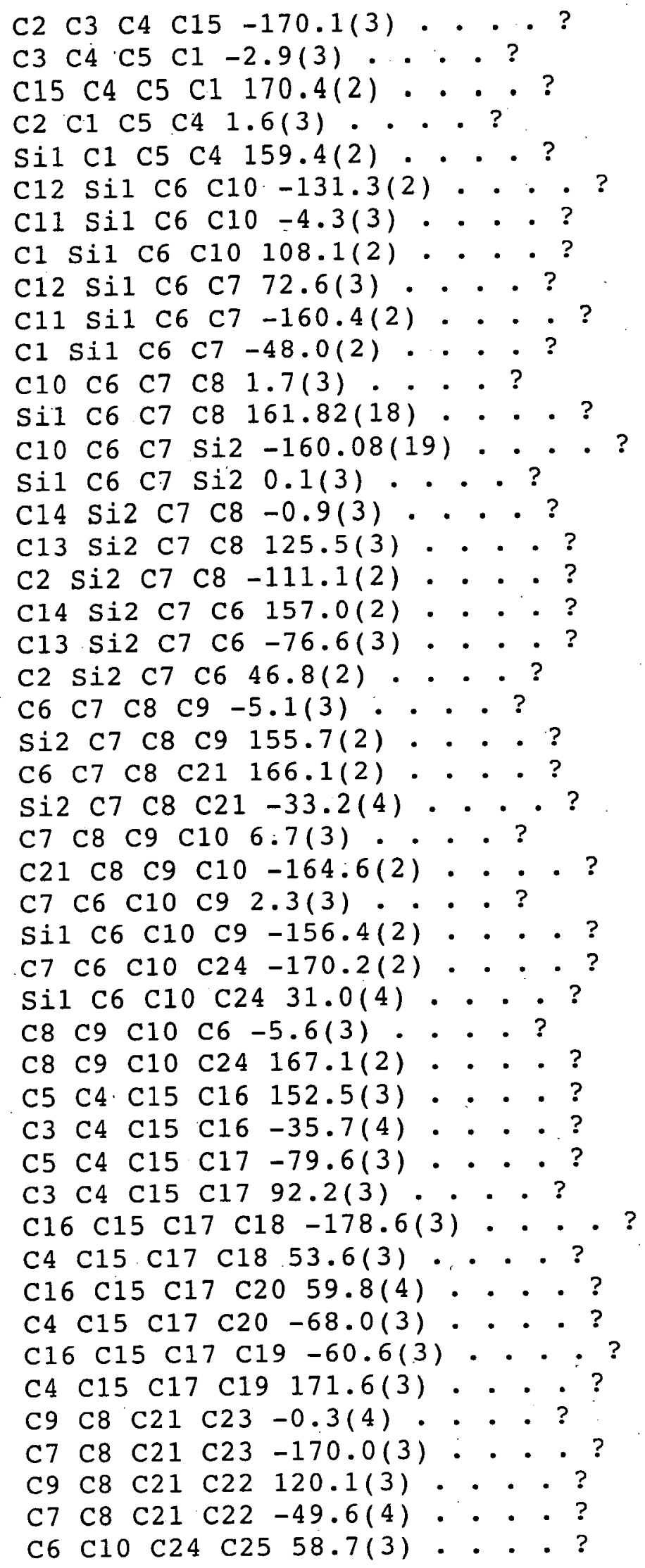


(C) 2004 American Chemical Society, J. Am. Chem. Soc., Baar ja040021j Supporting Info Page 34

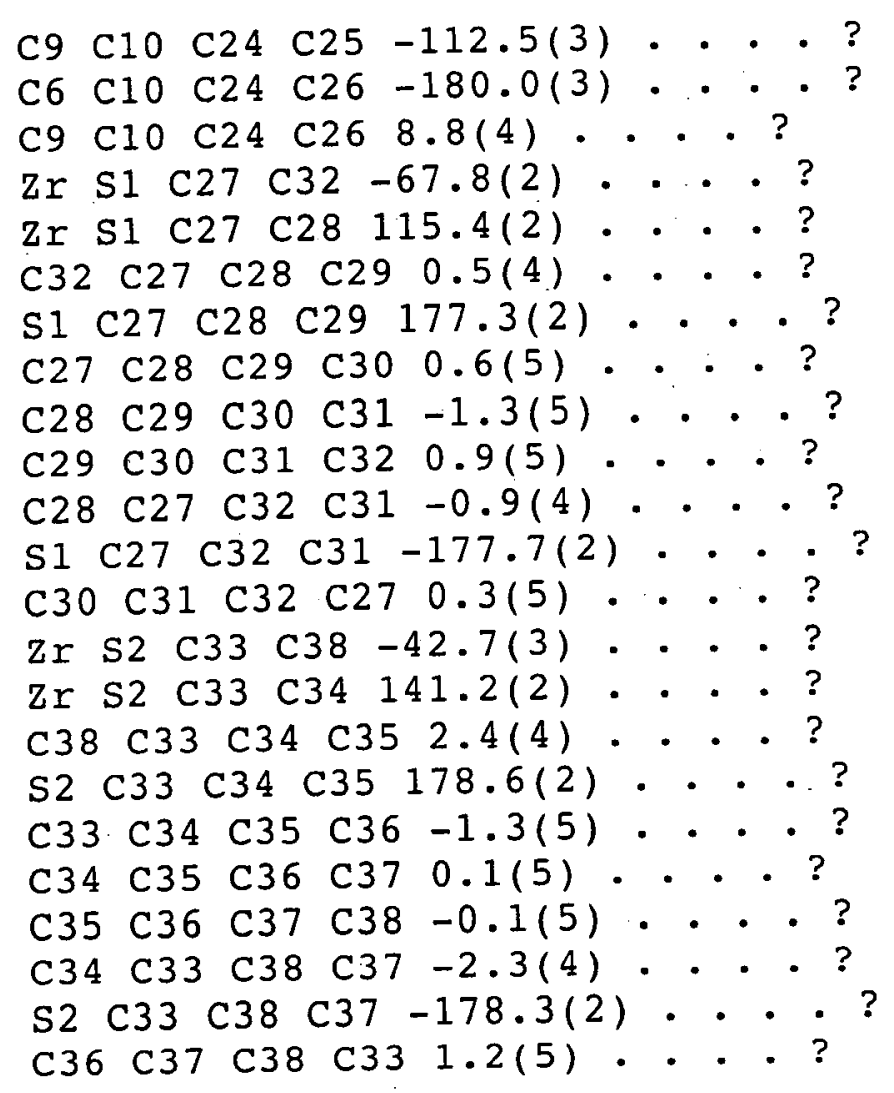

$\#===$ END 
(C) 2004 American Chemical Society, J. Am. Chem. Soc., Baar ja040021j Supporting Info Page 35

CIF FILE FOR ( $S)-6$

data_eymo1

audit_creation_method

SHELXL-9 7

-chemical_name_systematic

;

$?$

;

_chemical_name_common

?

chemical_melting_point

?

chemical_formula_moiety

?

chemical_formula_sum

'C33.50 $\mathrm{H} 54 \mathrm{Cl} 2 . \overline{\mathrm{S}} \mathrm{i} 2 \mathrm{Zr}$ '

_chemical_formula_weight

675.07

loop

_atom_type_symbol

atom_type_description

-atom_type_scat_dispersion_real

-atom_type_scat_dispersion_imag

-atom_type_scat_source

' $C$ ' ${ }^{\prime} \mathrm{C}{ }^{\prime}-0.00 \overline{3} 30.0016$

'International Tables Vol $\mathrm{C}$ Tables 4.2 .6 .8 and 6.1.1.4'

' $\mathrm{H}^{\prime} \quad \mathrm{H}^{\prime} \quad 0.0000 \quad 0.0000$

'International Tables Vol C Tables 4.2 .6 .8 and 6.1.1.4'

'Si' 'Si' $0.0817 \quad 0.0704$

'International Tables Vol C Tables 4.2 .6 .8 and 6.1.1.4'

' $\mathrm{Cl}$ ' ' $\mathrm{Cl}$ ' 0.14840 .1585

'International Tables Vol. C Tables 4.2 .6 .8 and 6.1.1.4'

' $z r$ ' ' $z r$ ' $-2.9673 \quad 0.5597$

'International Tables Vol C Tables 4.2 .6 .8 and 6.1.1.4'

symmetry_cell_setting

_symmetry_space_group_name_H-M

loop

_symmetry_equiv_pos_as_xyz

$' x, y, z '$
' $x,-y,-z '$

_cell_length_a

-cell_length_b

_cell_length_c

_cell_angle_alpha

-cell_angle_beta

_cell_angle_gamma

_cell_volume
Triclinic

P-1
$10.3602(5)$

$13.1543(7)$

$14.0843(7)$

$97.1990(10)$

$104.0680(10)$

$106.2750(10)$

$1748.26(15)$ 
(C) 2004 American Chemical Society, J. Am. Chem. Soc., Baar ja040021j Supporting Info Page 36

cell_formula_units_z

cell-measurement_temperature

_cell_measurement_reflns_used cell_measurement_theta_min

_cell_measurement_theta_max

_exptl_crystal_description

-exptl_crystal_colour

-expt1_crystal_size_max

-exptl_crystal_size_mid

-expt1_crystal_size_min

-exptl_crystal_density_meas

-exptl_crystal_density_diffrn

-expt1_crystal_density_method

-expt1 crystal_F_000

_exptI_absorpt_coefficient_mu

-exptl_absorpt_correction_type

-exptl_absorpt_correction_T_min

-exptl_absorpt_correction_T_max

_exptI_absorpt_process_detaīls

_exptl_special_details

;

?

;

diffrn_ambient_temperature

diffrn_radiation_wavelength

diffrn_radiation_type

diffrn_radiation_source

diffrn_radiation_monochromator

_diffrn_measurement_device_type

-diffrn_measurement_method

diffrn_detector_area_resol_mean

_diffrn_standards_number

diffrn_standards_interval_count

_diffrn_standards_interval_time

diffrn_standards_decay_o

diffrn_reflns_number

diffrn_reflns_av_R_equivalents

_diffrn_reflns_av_sigmaI/netI

_diffrn_reflns_limit_h_min

-diffrn_reflns_limit_h_max

_diffrn_reflns_limit_k_min

diffrn_reflns_limit_k_max

diffrn_reflns_limit_l_min

_diffrn_reflns_limit_l_max

_diffrn_reflns_theta_min
2

$100(2)$

24577

2.19

28.17

Plates

Colorless

0.24

0.22

0.15

?

1.282

'not measured'

714

0.557

none

0.8780

0.9212

?

$100(2)$

0.71073

MoK`a

'normal-focus sealed tube'

graphite

'Bruker SMART 1000'

' 'W scans at 7 If settings'

?

?

?

$?$

?

39577

0.0467

0.0310

$-13$

13

$-17$

17

$-17$

18

1.65 
(C) 2004 American Chemical Society, J. Am. Chem. Soc., Baar ja040021j Supporting Info Page 37

\begin{tabular}{|c|c|}
\hline $\begin{array}{l}\text { diffrn_reflns_theta_max } \\
\text { reflns_number_total } \\
\text { reflns_number_gt } \\
\text { reflns_threshold_expression }\end{array}$ & $\begin{array}{l}28.18 \\
7898 \\
7088 \\
>2 \mathrm{sigma}(\mathrm{I})\end{array}$ \\
\hline $\begin{array}{l}\text { computing_data_collection } \\
\text { computing_cell_refinement } \\
\text { computing_data_reduction } \\
\text { computing_structure_solution } \\
\text { computing_structure_refinement } \\
\text { computing_molecular_graphics } \\
\text { computing publication_material }\end{array}$ & $\begin{array}{l}\text { 'Bruker SMART v5.054' } \\
\text { 'Bruker SAINT v6.022', } \\
\text { 'Bruker SAINT v6.022' } \\
\text { 'SHELXS-97 (Sheldrick, 1990)' } \\
\text { 'SHELXL-97 (Sheldrick, 1997)' } \\
\text { 'Bruker SHELXTL' } \\
\text { 'Bruker SHELXTL' }\end{array}$ \\
\hline
\end{tabular}

$\bar{i}^{\text {refine_special_details }}$

Refinement of $F^{\wedge} 2^{\wedge}$ against ALL reflections. The weighted $R$-factor $W R$ and goodness of fit $S$ are based on $F^{\wedge} 2^{\wedge}$, conventional $R$-factors $R$ are based on $F$, with $F$ set to zero for negative $F^{\wedge} 2^{\wedge}$. The threshold expression of $F^{\wedge} 2^{\wedge}>2$ sigma $\left(F^{\wedge} 2^{\wedge}\right)$ is used only for calculating R-factors $(g t)$ etc. and is

not relevant to the choice of reflections for refinement. R-factors based

on $\mathrm{F}^{\wedge} 2^{\wedge}$ are statistically about twice as large as those based on $\mathrm{F}$, and R-

factors based on ALL data will be even larger.

;

refine_1s_structure_factor_coef _refine_ls_matrix_type refine_ls_weighting_scheme refine.ls_weighting_details atom sites solution primary -atom_sites_solution_secondary atom_sites_solution_hydrogens refine_ls_hydrogen_treatment -refine_ls_extinction_method refine_ls_extinction_coef -refine_ls_number_reflns refine_ls_number_parameters refine Is number restraints refine_ls_R_factor_all refine_ls_R_factor_gt -refine_ls_wR_factor_ref _refine_ls_wR_factor_gt -refine_Is_goodness_of_fit_ref -refine_ls_restrained_s_ali _refine_ls_shift/su_max _refine_ls_shift/su_mean

Fsqd

'Full matrix'

Sigma $' w=1 / s^{\wedge} 2^{\wedge}\left(F O^{\wedge} 2^{\wedge}\right)^{\prime}$

'Patterson method'

'Difference Fourier map'

'Geometric positions'

Riding

none

?

7898

398

0

0.0459

0.0407

0.0913

0.0909

2.923

2.923

0.002

0.000 
(C) 2004 American Chemical Society, J. Am. Chem. Soc., Baar ja040021j Supporting Info Page 38

loop_

atō_site_label

-atom_site_type_symbol

_atom_site_fract_x

-atom_site_fract_y

atom_site_fract_z

atom_site_u_iso_or_equiv

atom_site_adp_type

atom_site_occupancy

_atom_site_symmetry_multiplicity

atom_site_calc_flag

_atom_site_refiñement_flags

atom_site_disorder_assembly

atom_site_disorder_group

$\mathrm{z} \bar{r} 1 \mathrm{Zr} 0.61 \overline{6} 02(3) \quad 0 . \overline{799590(19)} 0.741901(18) 0.01144(8)$ Uani $11 \mathrm{~d} \cdot . \cdot$

Cl1 Cl $0.86464(7) \quad 0.82206(5) \quad 0.76355(5) \quad 0.01779(15)$ Uani $11 d .$.

Cl2 Cl $0.52234(7) \quad 0.75491(6) \quad 0.56124(5) \quad 0.02344(16)$ Uani $11 d$. .

Si1 Si $0.65652(8) \quad 0.84717(6) \quad 0.97679(5) \quad 0.01498(17)$ Uani $11 d . \quad$.

Si2 Si $0.33140(8) \quad 0.79150(6) \quad 0.80175(5) \quad 0.01560(17)$ Uani $11 d$. .

C1 C $0.6232(3) 0.9323(2) 0.88196(19) 0.0150(6)$ Uani $11 d$. . .

C2 C $0.4877(3) 0.9075(2) \quad 0.80696(19) \quad 0.0148(6)$ Uani 11 d. .

C3 C $0.5154(3) \quad 0.9557(2) \quad 0.7256(2) \quad 0.0158(6)$ Uani 11 d . .

H3 H $0.4459 \quad 0.95150 .6657 \quad 0.019$ Uiso 1 l calc $R$. .

C4 C $0.6601(3) 1.0104(2) 0.74672(19) \quad 0.0148(6)$ Uani 11 d. A.

C5 C $0.7263(3) \quad 0.99516(19) \quad 0.84199(19) 0.0146(6)$ Uani 1 l d . .

H5 H $0.8245 \quad 1.0226 \quad 0.8746 \quad 0.018$ Uiso 1 1 calc R . .

C6 C $0.5732(3) \quad 0.7169(2) 0.87941(18) 0.0131(5)$ Uani $11 \mathrm{~d}$. . .

C7 C $0.4353(3) \quad 0.6941(2) 0.80510(19) 0.0142(6)$ Uani 11 d . .

C8 C $0.4264(3) \quad 0.61269(19) 0.72402(18) 0.0136(5)$ Uani $11 d$.

C9 C $0.5536(3) \quad 0.5894(2) \quad 0.74464(19) \quad 0.0149(6)$ Uani 11 d . .

H9 H $0.5770 \quad 0.54150 .7006 \quad 0.018$ Uiso 1 l calc $\mathrm{R}$. .

C10 C $0.6412(3) \quad 0.6479(2) \quad 0.84065(19) \quad 0.0146(6)$ Uani 11 d...

C11 C $0.8494(3) \quad 0.8849(2) \quad 1.0339(2) \quad 0.0211(6)$ Uani 11 d . . .

H11A H $0.88490 .9577 \quad 1.07610 .032$ Uiso 11 calc $R$. .

H11B H $0.8956 \quad 0.8837 \quad 0.98100 .032$ Uiso 1 l calc R . .

H $11 \mathrm{C} \mathrm{H} 0.8695 \quad 0.8330 \quad 1.0749 .0 .032$ Uiso 1 l calc $\mathrm{R}$..

C12 C $0.5764(3) \quad 0.8525(2) 1.0815(2) 0.0236(7)$ Uani 11 . . .

H12A H 0.59380 .79831 .12020 .035 Uiso 11 calc $R$ • •

H12B H 0.47490 .83721 .05430 .035 Uiso 11 calc $R$. .

$\mathrm{H} 12 \mathrm{C} \mathrm{H} \quad 0.6184 \quad 0.9247 \quad 1.1250 \quad 0.035$ Uiso 11 calc $\mathrm{R}$.

C13 C $0.2552(3) \quad 0.8102(2) \quad 0.9074(2) \quad 0.0242(7)$ Uani 11 d .

H13A H 0.15770 .80830 .88090 .036 Uiso 11 calc $R$. .

H13B H $0.3104 \quad 0.88020 .95250 .036$ Uiso 11 calc $R$. .

$\mathrm{H} 13 \mathrm{C} \mathrm{H} \quad 0.2575 \quad 0.7520 \quad 0.94420 .036$ Uiso 1 calc $\mathrm{R}$. .

C14 C 0.1932 (3) $0.7702(2) \quad 0.6814(2) \quad 0.0246(7) \quad \operatorname{Uani} 11 \mathrm{~d}$. .

H14A H $0.11690 .7026 \quad 0.6727 \quad 0.037$ Uiso 11 calc $R$. .

$\mathrm{H} 14 \mathrm{~B}$ H $0.23410 .7657 \quad 0.6258 \quad 0.037$ Uiso 1 l calc $\mathrm{R}$. . 
H $14 \mathrm{C}$ H 0.15590 .83090 .68250 .037 Uiso 1 I calc $R$. . C15 C $0.7289(3) 1.08001(19) 0.68260(18) 0.0177(6)$ Uani $11 \mathrm{~d} .$. $\begin{array}{llllllllll}\mathrm{H} 15 \mathrm{~A} \mathrm{H} & 0.8269 & 1.0772 & 0.6947 & 0.021 & \text { Uiso } 0.50 & 1 & \text { calc } \mathrm{PR} \text { A } 1\end{array}$ H15B H 0.66991 .05360 .61330 .021 Uiso $0.501 \mathrm{~d}$ PR A 2 C16A C $0.6540(3) 1.03794(19) 0.56919(18) 0.0236(13)$ Uani 0.501 d PR A 1 H16A H $0.7150 \quad 1.0730 \quad 0.53130 .035$ Uiso 0.501 calc PR A 1 H16B H $0.56591 .0548 \quad 0.55210 .035$ Uiso 0.501 calc PR A 1 H16C H $0.6336 \quad 0.9594 \quad 0.55240 .035$ Uiso 0.501 calc PR A 1 C16B C $0.8719(6) 1.0736(5) 0.6874(5) 0.0279(15)$ Uani 0.501 d P A 2 H16D H $0.9373 \quad 1.1127 \quad 0.75310 .042$ Uiso 0.501 calc PR A 2 $\begin{array}{llllllllll}\mathrm{H} 16 \mathrm{E} & \mathrm{H} & 0.9042 & 1.1064 & 0.6350 & 0.042 & \text { Uiso } 0.50 & 1 & \text { calc PR A } 2\end{array}$

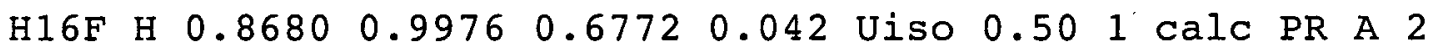
C17 C $0.7401(3) 1.2011(2) 0.7156(2) 0.0232(7)$ Uani 11 d. A. C18 C $0.8335(4) 1.2467(2) \quad 0.8239(2) \quad 0.0450(10)$ Uani $11 \mathrm{~d} .$. H18A H $0.8434 \quad 1.32350 .84240 .068$ Uiso 1 l calc $R A$. H18B H 0.9264 .1 .23910 .82990 .068 Uiso 1 l calc R . . H18C H $0.7903 \quad 1.2068 \quad 0.86860 .068$ Uiso 1 calc R . C19 C $0.8071(3) 1.2677(2) 0.6473(2) 0.0306(8)$ Uani 11 d . . H19A H 0.74791 .24000 .57780 .046 Uiso 1 l calc $R$ A. H19B H $0.9007 \quad 1.26160 .6527 \quad 0.046$ Uiso 1 1 calc R . H19C H 0.81551 .34380 .66790 .046 Uiso 1 l calc R. . C20 C $0.5963(4) 1.2128(3) 0.7065(2) 0.0339(8)$ Uani 11 d . . H20A H $0.6062 \quad 1.2895 \quad 0.7251 \quad 0.051$ Uiso 11 calc $R A$. H20B H $0.55331 .1728 \quad 0.75120 .051$ Uiso 11 calc $R$. . H20C H $0.5366 \quad 1.1837 \quad 0.63720 .051$ Uiso 11 calc R. . C21 C $0.2971(3) \quad 0.5507(2) 0.6368(2) \quad 0.0170(6)$ Uani $11 d .$. H21 H $0.2627 \quad 0.6049 \quad 0.60350 .020$ Uiso 1 l calc R . C22 C $0.1818(3) 0.4884(2) 0.6792(2) 0.0232(7)$ Uani 11 d . . H22A H 0.16130 .54110 .72510 .028 Uiso 1 l calc $R$. . H22B H 0.09490 .45180 .62330 .028 Uiso 11 calc $R$. C23 C $0.2196(4) 0.4046(3) 0.7351(3) 0.0390(9)$ Uani $11 d . .$. H23A H 0.21910 .34340 .68740 .059 Uiso 1 l calc $R$. • H23B H $0.1506 \quad 0.3791 \quad 0.77090 .059$ Uiso 1 l calc $R$. H23C н $0.3134 \quad 0.43720 .78320 .059$ Uiso 1 l calc $\mathrm{R}$. C24 C $0.3283(3) 0.4769$ (2) $0.5581(2) 0.0220(6)$ Uani $11 \mathrm{~d}$. . $\mathrm{H} 24 \mathrm{~A} \mathrm{H} \quad 0.40730 .52050 .53730 .026$ Uiso 1 l calc $\mathrm{R}$. H24B H $0.35880 .42070 .5890 \quad 0.026$ Uiso 11 calc R. C25 C 0.2035 (3) $0.4216(3) 0.4657(2) 0.0316(8)$ Uani $11 \mathrm{~d}$. . H25A H 0.12730 .37360 .48480 .047 Uiso 11 calc $R$. . $\begin{array}{lllllllllll}\mathrm{H} 25 \mathrm{~B} & \mathrm{H} & 0.2319 & 0.3791 & 0.4172 & 0.047 & \text { Uiso } 1 & 1 & \text { calc } \mathrm{R} & \text {. }\end{array}$ H25C H $0.1708 \quad 0.47650 .4356 \quad 0.047$ Uiso 1 l calc $\mathrm{R}$. . C26 C $0.7767(3) 0.6306(2) 0.89542(19) 0.0157(6)$ Uani 11 d . . $\mathrm{H} 26 \mathrm{H} \quad 0.8501 \quad 0.7035 \quad 0.92210 .019$ Uiso 11 calc $\mathrm{R}$. C27 C $0.7531(3) 0.5809(2) 0.9860(2) 0.0205(6)$ Uani 11 d... H27A H $0.84490 .5807 \quad 1.02790 .025$ Uiso 11 calc $R$. . H27B H $0.71860 .6281 \quad 1.02690 .025$ Uiso 11 calc $R$. C28 C $0.6493(3) 0.4654(2) 0.9597(2) 0.0271(7)$ Uani $11 d .$. H28A H $0.5597 \quad 0.4633 \quad 0.9146 \quad 0.041$ Uiso 1 l calc. R. 
H28B H $0.63410 .4432 \quad 1.02100 .041$ Uiso 11 calc $R$. H28C H $0.68790 .4160 \quad 0.9266 \quad 0.041$ Uiso 11 calc R. . C29 C $0.8292(3) \quad 0.5644(2) 0.8252(2) \quad 0.0195(6)$ Uani $11 d$. . H29A H 0.75770 .49200 .79740 .023 Uiso 1 l calc R. • H29B H $0.8397 \quad 0.60050 .76860 .023$ Uiso 1 l calc $R$ C30 C $0.9680(3) \quad 0.5507(2) 0.8759(2) 0.0283(7)$ Uani $11 d . \cdots$ H $30 \mathrm{~A}$ H $1.03720 .62190 .9092 \quad 0.042$ Uiso 11 calc $R$. H3OB H $1.00150 .51550 .8258 \quad 0.042$ Uiso 11 calc $R$. $\begin{array}{lllllllll}\text { H } 30 \text { H } & 0.9553 & 0.5058 & 0.9256 & 0.042 & \text { Uiso } 1 & 1 & \text { calc } R \text {. }\end{array}$ C41 C $-0.1747(8)-0.0594(8) 0.3929(6) \quad 0.044(2)$ Uani 0.501 d P . H41A H $-0.2317-0.12150 .4100 \quad 0.066$ Uiso $0.501 \mathrm{~d}$ PR . H41B H $-0.2295-0.01350 .37330 .066$ Uiso 0.501 d PR . . H4 IC H $-0.1421-0.0827 \quad 0.33840 .066$ Uiso $0.501 \mathrm{~d}$ PR . C42 C $-0.0506(6) \quad 0.0024(5) \quad 0.4822(5) \quad 0.0286(14)$ Uani $0.501 \mathrm{~d} P$. C43 C $0.0465(7) \quad 0.0949(5) \quad 0.4767(5) \quad 0.0312(16)$ Uani 0.501 d P . . H43A H $0.0318 \quad 0.12260 .41650 .037$ Uiso $0.501 \mathrm{~d}$ PR . C44C $0.1658(7) 0.1495(5) 0.5560(5) 0.0310(15)$ Uani $0.501 \mathrm{~d} P$.

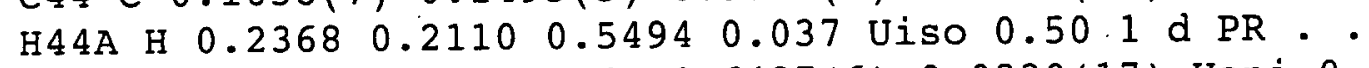
C45 C $0.1842(8) \quad 0.1151(6) \quad 0.6437(6) \quad 0.0330(17)$ Uani 0.501 d P.. H45A H $0.2635 \quad 0.15550 .70020 .040$ Uiso 0.501 d PR . . C46 C $0.0869(8) 0.0217(5) \quad 0.6529(5) \quad 0.0359(16)$ Uani 0.501 d P.. H46A H $0.1008-0.0038 \quad 0.7143 \quad 0.043$ Uiso 0.501 d PR . C47 C $-0.0308(7)-0.0329(6) 0.5703(5) 0.0311(15)$ Uani $0.501 \mathrm{~d}$ P. H47A H $-0.0991-0.09690 .57550 .037$ Uiso $0.501 \mathrm{~d} P R$.

\section{loop}

-atom_site_aniso_label
-atom_site_aniso_U_11
-atom_site_aniso_U_22
-atom_site_aniso_U_33
-atom_site_aniso_U_23
-atom_site_aniso_U_13
-atom_site_aniso_U_12
-atom_site-anis)

$\mathrm{z} \overline{\mathrm{r}} 10 . \overline{0} 1265(14) \quad 0.0 \overline{1} 060(13) \quad 0.01110(13) \quad 0.00190(9) \quad 0.00344(10) \quad 0.00402(10)$

Cl1 $0.0156(3) \quad 0.0183(3) \quad 0.0213(3) \quad 0.0059(3) \quad 0.0070(3) \quad 0.0060(3)$

Cl2 $0.0264(4) \quad 0.0282(4) \quad 0.0133(3) \quad 0.0028(3) \quad 0.0032(3) \quad 0.0080(3)$

Si1 $0.0173(4) \quad 0.0136(4) \quad 0.0122(4) \quad 0.0008(3) 0.0038(3) 0.0033(3)$

Si2 $0.0141(4) \quad 0.0137(4) \quad 0.0190(4) \quad 0.0014(3) \quad 0.0059(3) \quad 0.0046(3)$

$\mathrm{C} 10.0185(15) \quad 0.0113(13) \quad 0.0134(13)-0.0009(10) 0.0047(11) 0.0036(11)$

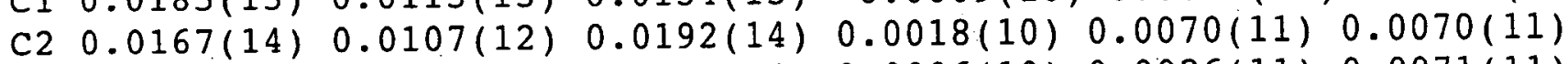

C3 $0.0182(15) \quad 0.0114(13) \quad 0.0172(14) 0.0006(10) \quad 0.0026(11) 0.0071(11)$

$\begin{array}{lllllll}\text { C4 } & 0.0171(14) & 0.0108(13) & 0.0175(14) & 0.0014(10) & 0.0056(11) & 0.0063(11)\end{array}$

C5 $0.0164(14) \quad 0.0094(12) 0.0158(13)-0.0022(10) 0.0035(11) 0.0036(11)$

C6 $0.0157(14) 0.0122(13) 0.0134(13) 0.0051(10) 0.0067(11) 0.0047(11)$

C7 $0.0126(14) 0.0134(13) 0.0152(13) 0.0030(10) \cdot 0.0057(11) 0.0008(11)$

C8 $0.0159(14) \quad 0.0105(12) \quad 0.0141(13) \quad 0.0026(10) \quad 0.0059(11) 0.0024(11)$

C9 $0.0187(14) \quad 0.0108(12) 0.0162(14) 0.0020(10) 0.0077(11) 0.0044(11)$

C10 $0.0171(14) \quad 0.0109(12) \quad 0.0160(13) 0.0053(10) 0.0067(11) 0.0024(11)$ 